\title{
Espécies da flora nativa nas Estações Experimentais da Agência Paulista de Tecnologia dos Agronegócios, Instituto Agronômico de Campinas, Estado de São Paulo, Brasil
}

\author{
Ana Cláudia Oliveira de Souza ${ }^{1,3}$, Roseli Buzanelli Torres² ${ }^{2}$ Luís Carlos Bernacci² e \\ Sigrid Luiza Jung-Mendaçolli²
}

Recebido: 27.05.2014; aceito: 12.09.2014

\begin{abstract}
Species of native flora of the remnants from Experimental Stations of Agência Paulista de Tecnologia dos Agronegócios, Instituto Agronômico de Campinas, São Paulo State, Brazil). The native vegetation of the state of São Paulo is mainly characterized by small isolated fragments, like the ones found in several Agência Paulista de Tecnologia dos Agronegócios experimental stations. Based on exsiccates from the Instituto Agronômico de Campinas Herbarium collection, we elaborated a list of the vascular flora of these remnants in 21 experimental stations. Mata Atlântica remnants are predominant, but three experimental stations had also fragments of Cerrado, and only two had no species recorded in the collection. We found 2,457 samples belonging to 120 families, 493 genera, and 947 species. The most diverse families were Fabaceae, Asteraceae, and Rubiaceae. Two species are threatened of extinction. Taxa with medicinal, ornamental, and/or feeding potential were found, as well as species not reported for the state of São Paulo in the Flora of Brasil database. The results indicate the need of regularization of the legal and permanent conservation areas and the need to increase the mechanisms of protection of the remnants and their rich genetic inheritance.
\end{abstract}

Keywords: biodiversity, in situ conservation, native germplasm, threatened species

RESUMO - (Espécies da flora nativa nas Estações Experimentais da Agência Paulista de Tecnologia dos Agronegócios, Instituto Agronômico de Campinas, Estado de São Paulo, Brasil). A vegetação nativa do Estado de São Paulo está representada, principalmente, por pequenos e isolados fragmentos, como os que ocorrem em várias Estações Experimentais da Agência Paulista de Tecnologia dos Agronegócios. Com base nas exsicatas do acervo do Herbário do Instituto Agronômico de Campinas, elaborou-se a listagem da flora vascular desses remanescentes em 21 Estações Experimentais. Predominam remanescentes de Mata Atlântica, mas três unidades possuem também fragmentos de Cerrado, e apenas duas não têm nenhum registro de espécies no acervo. Foram encontrados 2.457 materiais distribuídos em 120 famílias, 493 gêneros e 947 espécies. As famílias mais ricas foram Fabaceae, Asteraceae e Rubiaceae, observando-se duas espécies ameaçadas de extinção, táxons com potencial medicinal, ornamental e/ou alimentício e espécies não citadas para o Estado de São Paulo na Lista de Espécies da Flora do Brasil. Os resultados indicam a necessidade de regularização das áreas de reserva legal e de preservação permanente, e a necessidade de aumentar os mecanismos de proteção dos remanescentes e seu rico patrimônio genético. Palavras-chave: biodiversidade, conservação in situ, espécies ameaçadas, germoplasma nativo

\section{Introdução}

Por volta da segunda metade do século XVII, os registros de desmatamento eram ainda de pequena escala nas cercanias da cidade de São Paulo, e até a metade do século XIX, a vegetação nativa do Estado era relativamente bem conservada. A partir da expansão da cultura cafeeira, o ritmo de desmatamento acelerou de modo avassalador (Dean 1996, Victor et al. 2005). Segundo Victor et al. (2005), quase $82 \%$ do território paulista era coberto por formações florestais, predominando o Domínio da Mata Atlântica, com penínsulas e áreas disjuntas periféricas de Cerrado (Durigan et al. 2004), em suas diferentes fisionomias.

1. Programa de Pós-Graduação do Instituto Agronômico de Campinas, Agricultura Tropical e Subtropical, Gestão de Recursos Agroambientais

2. Instituto Agronômico de Campinas, Núcleo de P\&D Jardim Botânico, Av. Theodureto de A. Camargo, 1.500, Vila Nova, 13075-630 Campinas, SP, Brasil

3. Autor para correspondência: bio.anaclaudia@gmail.com 
A Floresta Atlântica e o Cerrado são considerados áreas prioritárias para a conservação da biodiversidade no mundo (hotspots) e apresentam altas taxas de endemismo, 39,5\% e 33,3\%, respectivamente (Forzza et al. 2012). Os remanescentes da Mata Atlântica e do Cerrado, que representam aproximadamente $14 \%$ da área de São Paulo (FAPESP 2006), encontram-se em geral na forma de pequenos fragmentos, altamente perturbados, isolados, pouco conhecidos e pouco protegidos, especialmente em paisagens intensamente cultivadas (Viana 1995).

No processo de alteração do uso do solo a vegetação nativa pode ser totalmente eliminada ou fragmentada, o que resulta em pequenas áreas residuais e isoladas de remanescentes (Olifiers \& Cerqueira 2006). A fragmentação em grande escala causa diversas alterações nos processos biológicos e nas interações entre os organismos, além de resultar na perda gradual da diversidade biológica (Cerqueira et al. 2003), afetando especialmente a biodiversidade regional (Almeida 1998). A drástica redução das populações locais, decorrente da fragmentação dos ambientes, afeta negativamente os três níveis de organização da diversidade biológica - intraespecífica, interespecífica e nos ecossistemas.

Embora seja um país megadiverso, a agricultura no Brasil apóia-se num pequeno número de espécies exóticas domesticadas. Dentre as 15 plantas mais importantes que são cultivadas no mundo, apenas o amendoim e a mandioca são nativos do Brasil, o que configura grande vulnerabilidade alimentar. Para minimizar a perda genética decorrente dos efeitos da síndrome de domesticação que ocorre nas espécies cultivadas (Nass \& Sigrist 2009, Flint-Garcia 2013), trabalhos de melhoramento genético podem ser desenvolvidos com a utilização de parentes silvestres e variedades crioulas (Walter et al. 2005a,b).

No âmbito da Convenção da Diversidade Biológica (CDB), foi editado o Decreto número 4.339/2002 que, dentre as suas várias diretrizes preconiza o conhecimento e a conservação da biodiversidade. Para tanto, estabelece diretrizes para a conservação in situ e ex situ da variabilidade genética, de ecossistemas, incluindo os serviços ambientais, e das espécies, particularmente das ameaçadas ou com potencial econômico (Leite \& Coradin 2011).

Considerando o quadro de extensa degradação ambiental e da fragmentação da vegetação nativa do Estado de São Paulo, ressalta-se a importância dos remanescentes de Mata Atlântica e de Cerrado existentes nas Estações Experimentais (EEx) do
Instituto Agronômico (IAC) pelo seu valor biológico intrínseco, pelos serviços ambientais que fornecem, como polinização, inimigos naturais de pragas e doenças de culturas agrícolas, preservação dos recursos hídricos, e pelo seu potencial econômico a ser pesquisado.

Os objetivos do presente trabalho foram analisar as espécies nativas encontradas nos remanescentes de vegetação das Estações Experimentais da Agência Paulista de Tecnologia dos Agronegócios/Instituto Agronômico de Campinas, identificando a ocorrência de espécies ameaçadas, aquelas com potencial agronômico e/ou medicinal, tendo como base os materiais botânicos depositados no Herbário do Instituto Agronômico de Campinas(IAC).

\section{Material e métodos}

As Estações Experimentais do Instituto Agrômico de Campinas - No contexto da expansão da cafeicultura no Estado de São Paulo, em 1887, Dom Pedro II fundou a Imperial Estação Agronômica de Campinas, que adquiriu maior relevância científica a partir de 1920, já então como Instituto Agronômico (IAC). Nesse período, o IAC consolidou um amplo programa de pesquisas com a criação das Divisões de Genética (1927) e de Solos (1935) (Santos 2005). Ao mesmo tempo, foram instaladas as Estações Experimentais (EEx) em diferentes municípios: Tietê (1924 - para estudos com algodão e feijão), São Roque (1927 viticultura), Limeira (1928 - citricultura), Piracicaba (1930 - cana-de-açúcar), Ubatuba (1931 - cacau, canade-açúcar e seringueira), Tatuí (1933 - algodão, arroz e milho), Pindorama (1934 - café, milho e algodão), Ribeirão Preto (1934 - cana-de-açúcar, café e soja), Jundiaí (1936 - uva de mesa e outras frutas de clima temperado), Mococa (1939 - citricultura), Monte Alegre do Sul (1942 - hortaliças e frutas de clima temperado) (Capozzoli 2009). Em 2002, foi criada a Agência Paulista de Tecnologia dos Agronegócios (APTA) com o objetivo de reorganizar os Institutos de Pesquisa da Secretaria de Agricultura e Abastecimento, e a maioria das EEx do IAC foram transformadas em Pólos Regionais de Desenvolvimento.

Para o presente estudo consideramos as 21 unidades que constituíam as EEx do IAC (tabela 1) antes da criação da APTA. Atualmente, o IAC tem áreas experimentais apenas em Campinas, Limeira/ Cordeirópolis, Jundiaí e Ribeirão Preto, estando as demais 17 vinculadas aos Pólos Regionais de Desenvolvimento. 
Levantamento e análise dos dados - A partir do banco de dados do Herbário IAC (http://herbario.iac.sp.gov. br/) foi elaborada a listagem das espécies nativas e naturalizadas coletadas nas EEx do Instituto; de posse dessa listagem, foram verificadas as informações das fichas de cada material, para confirmação do local de coleta. Para a elaboração da listagem, os nomes das espécies foram atualizados, consultando-se, principalmente, a Lista das Espécies da Flora do Brasil (http://reflora.jbrj.gov.br/), os volumes da Flora Fanerogâmica do Estado de São Paulo (Wanderley et al. 2001, 2002, 2003, 2005, 2007, 2009, 2012); e bancos de dados The Plant List (http://www. theplantlist.org/), Tropicos (http://www.tropicos.org/) e The International Plant Names Index (http://www. ipni.org/ipni/plantnamesearchpage.do). A ocorrência de espécies ameaçadas de extinção foi verificada na Resolução SMA-48 (Secretaria do Meio Ambiente do Estado de São Paulo 2004) e em Martinelli \& Moraes (2013). Consultando Walter et al. (2005a), Coradin et al. (2011) e Aguiar et al. (2014) verificamos a ocorrência de espécies com potencial econômico. Ao consultar a Lista de Espécies da Flora do Brasil, observou-se também a ocorrência da espécie no Estado de São Paulo.

\section{Resultados}

A maioria das EEx não possui informações precisas sobre o tamanho das áreas nativas que, em geral, não correspondem à reserva legal ou às áreas de preservação permanente (tabela 1). Alguns estudos botânicos ou ecológicos foram realizados em áreas nativas, como os de Torres et al. $(1992,1994)$, Rodrigues et al. (2004), Ferreira et al. (2007), Teixeira Neto (2009), Sampaio et al. (2012) e Carvalho et al. (2013), em Campinas; Tannus (2007) e Scaramuzza (2008), em Itararé; Silva \& Leitão Filho (1982), em Ubatuba; Ivanauskas et al. (2001), em Pariquera-Açu onde, segundo Sakai \& Lepsch (1984), 44\% da área é composta pelas várzeas do rio Jacupiranga. Quanto ao histórico dos remanescentes, algumas EEx dispõem de informações, como Jaú, Pindamonhangaba e Piracicaba, cujas áreas de matas são constituídas parcial ou totalmente por reflorestamento. Em Monte Alegre do Sul, São Roque e Votuporanga, a maior parte das áreas nativas é decorrente de regeneração natural, com cerca de 50 anos. Na EEx de Ribeirão Preto as áreas de preservação permanente estão muito degradadas e a reserva legal ainda precisa ser recomposta
$\mathrm{O}$ acervo da flora nativa das EEx da APTA/IAC no Herbário IAC está atualmente constituído por 2.457 exsicatas (figura 1) e os primeiros registros datam de 1936. Do total das 21 Estações Experimentais que pertenciam ao IAC, 19 têm registro no acervo, mas o número de amostras é bastante desigual entre elas. AEEx de Guaíra não tem área remanescente, Piracicaba não tem registro de coletas e as unidades de Jaú, Capão Bonito, Assis, Tatuí e Limeira apresentam menos de dez exsicatas cada uma. A maioria das coletas (aproximadamente 60\%) foi realizada nas EEx de Pariquera-Açu, Campinas, Monte Alegre do Sul e Jundiaí.

$\mathrm{O}$ estudo dos materiais revelou um total de 120 famílias, 493 gêneros e 947 espécies (tabela 2). Considerando-se a ocorrência de aproximadamente 7.500 espécies de fanerógamas no Estado de São Paulo (Wanderley et al. 2012), a quantidade de espécies encontradas nas EEx da APTA/IAC representa 12\% dessa estimativa. As cinco famílias que apresentaram maior número de espécies foram Fabaceae (101 spp.), Asteraceae (84), Rubiaceae (73), Myrtaceae (58) e Poaceae (50). Por outro lado, famílias com ampla ocorrência em regiões tropicais, como Orchidaceae, Bromeliaceae e Cactaceae foram pouco coletadas nas EEx, respectivamente com oito, sete e dois gêneros. Constatou-se a ocorrência de 14 espécies nativas no Estado de São Paulo que não estão assim mencionadas na Lista de Espécies da Flora do Brasil: Adenocalymma dusenii, Manihot carthaginensis, Melampodium divaricatum, Paepalanthus albo-vaginatus, Paepalanthus elongatus, Paspalum oteroi, Praxelis pauciflora, Poincianella pluviosa, Sambucus canadensis, Sabicea villosa, Scleria gaertneri, Stachytarpheta polyura, Stevia rebaudiana e Trigonia eriosperma.

Consultando Martinelli \& Moraes (2013) foram encontradas duas espécies ameaçadas de extinção nos remanescentes: Dalbergia nigra (vulnerável, Lima et al. 2013) e Nematanthus monanthos (em perigo, Chautems et al. 2013). Considerando a Resolução SMA-48 (Secretaria de Meio Ambiente de São Paulo 2004), teríamos 18 espécies em diferentes categorias de risco: Aiouea acarodomatifera, Cissampelos pareira, Coussarea nodosa, Dalbergia nigra, Gouania ulmifolia, Myracrodruon urundeuva, Myrcia flagellaris, Nectandra cissiflora, Nematanthus monanthos, Ocotea nectandrifolia, Paspalum compressifolium, Peperomia nitida, Psychotria capitata, Simira viridiflora (tabela 2).

A avaliação de espécies de interesse econômico identificou aproximadamente 50 gêneros com espécies de uso corrente ou potencial como medicinal, ornamental, alimentício, madeireiro, oleráceo ou 
forrageiro, (tabela 2). Na Relação Nacional de Plantas Medicinais de Interesse ao Sistema Único de Saúde (RENISUS) constam 71 espécies, das quais pelo menos 14 (aproximadamente 20\%) podem ser encontradas nos remanescentes de vegetação nativa da APTA/IAC. Espécies de interesse ornamental podem ser encontradas em famílias como Alstroemeriaceae, Amaryllidaceae e Araceae; e espécies de importância alimentícia consagrada, em gêneros como Ananas, Annona e Passiflora, dentre outros.
Em diversos remanescentes de vegetação nativa da APTA/IAC foi observada a ocorrência das espécies exóticas invasoras Leucaena leucocephala, Cenchrus ciliaris, Cynodon dactylon e Hyparrhenia rufa (tabela 2).

\section{Discussão}

Nas EEx encontram-se diferentes formações vegetacionais (tabela 1), de dois domínios

Tabela 1. Estações Experimentais da Agência Paulista de Tecnologia dos Agronegócios (APTA)/Instituto Agronômico de Campinas(IAC), Estado de São Paulo, Brasil. Tamanho das áreas de reserva legal atual e necessário, de acordo com o Código Florestal Brasileiro, e área dos remanescentes. Brej: brejo; Camp: campo; CAlt: campo altimontano; Cerr: cerrado; FESD: floresta estacional semidecídua; FHig: floresta higrófila; FODe: floresta ombrófila densa; MCil: mata ciliar; MSec: mata secundária; Varz: várzea.

Table 1. Experimental Stations of Agência Paulista de Tecnologia dos Agronegócios (APTA)/ Instituto Agronômico de Campinas (IAC), São Paulo State, Brazil. Current and necessary legal reserve areas, according to the Brazilian Forest Code, and the remaining areas. Brej: swamp; Camp: meadow; CAlt: upper montane meadow; Cerr: cerrado; FESD: semideciduous seasonal forest; FHig: hygrophilous forest; FODe: rain forest; MCil: gallery forest; MSec: secondary forest and Varz: floodplain.

\begin{tabular}{lrrrrr}
\hline & Tamanho da & \multicolumn{2}{c}{ Reserva Legal (ha) } & & \\
\cline { 3 - 4 } Estação Experimental & unidade (ha) & Existente & Necessária & Área nativa (ha) & Formação Vegetaconal \\
& & & & \\
Adamantina & 175,5 & 13,1 & 34,0 & $\sim 50,0$ & MCil \\
Assis & 359,5 & 201,5 & 71,9 & 120,0 & FESD \\
Campinas & 692,0 & 94,0 & 140,0 & 94,0 & FHig; Camp; Cerr; MCil; FESD; \\
Capão Bonito & 97,1 & 11,0 & 19,4 & 16,0 & Varz \\
Guaíra & 4,0 & & & 0 & Cerr; FESD \\
Itararé & 490,0 & 103,0 & 98,0 & 120,0 & \\
Jaú & 126,9 & 0 & 25,4 & $\sim 11,0$ & CAlt \\
Jundiaí & 142,8 & 35,0 & & 60,9 & FESD \\
Limeira/Cordeirópolis & 197,0 & & & $\sim 8,0$ & FESD; MSec \\
Mococa & 460,0 & 24,7 & 92,0 & 30,0 & FESD \\
Monte Alegre do Sul & 351,7 & 205,6 & 70,3 & 180,0 & FESD \\
Pariquera-Açu & 256,0 & 122,5 & 51,2 & 122,5 & Brej; FODe; MCil \\
Pindamonhangaba & $1.234,0$ & 175,7 & 246,8 & 80,0 & FESD \\
Pindorama & 513,0 & 127,9 & 102,6 & $\sim 120,0$ & FESD \\
Piracicaba & 253,1 & 5,0 & 50,6 & $\sim 20,0$ & FESD; MCil \\
Ribeirão Preto & 821,8 & 3,3 & 164,4 & 119,5 & MCil \\
São Roque & 43,6 & 10,0 & 8,7 & 7,4 & FESD; MSec \\
Tatuí & 117,4 & 7,0 & 23,5 & $\sim 7,5$ & FESD \\
Tietê & 253,8 & 90,0 & 50,8 & $\sim 60,0$ & FESD \\
Ubatuba & 424,6 & 390,7 & 84,9 & $\sim 390,0$ & FHig; FODe; MCil \\
Votuporanga & 425,0 & 131,0 & 85,0 & $\sim 200,0$ & Cerr; FESD; MCil \\
\hline & & & & &
\end{tabular}

${ }^{1} \mathrm{~A}$ área física da EEx pertencente à municipalidade 
fitogeográficos brasileiros altamente ameaçados, a Mata Atlântica e o Cerrado. A relevância de conservar esses remanescentes é grande, considerando-se que da área original da Mata Atlântica restam apenas 11\% no Brasil, proporção semelhante no Estado de São Paulo, e que essas áreas estão representadas por pequenos fragmentos florestais, a maioria menor que 100 ha (Stehmann et al. 2009). No nível da paisagem, esses remanescentes são fonte de sementes para a produção de mudas de espécies nativas para reflorestamento e funcionam como corredores para a interligação dos fragmentos. Em Campinas (Fazenda Santa Elisa), Capão Bonito e Votuporanga ocorrem remanescentes de Mata Atlântica e de Cerrado. O remanescente de Mata Atlântica da EEx de Campinas possui aproximadamente 15 ha e, apesar da abundância de indivíduos das fases iniciais da sucessão florestal, apresenta similaridade florística com outros remanescentes da área urbana do município (A.C.O. de Souza, comunicação pessoal). A área de Cerrado tem particular importância devido à sua biodiversidade (Carvalho et al. 2013) e em função de ser um dos poucos remanescentes deste domínio no município de Campinas.

Considerando-se que mesmo as áreas mais estudadas necessitam de maior esforço de coleta, estima-se o que o número de espécies encontradas

Número de coletas por EEx

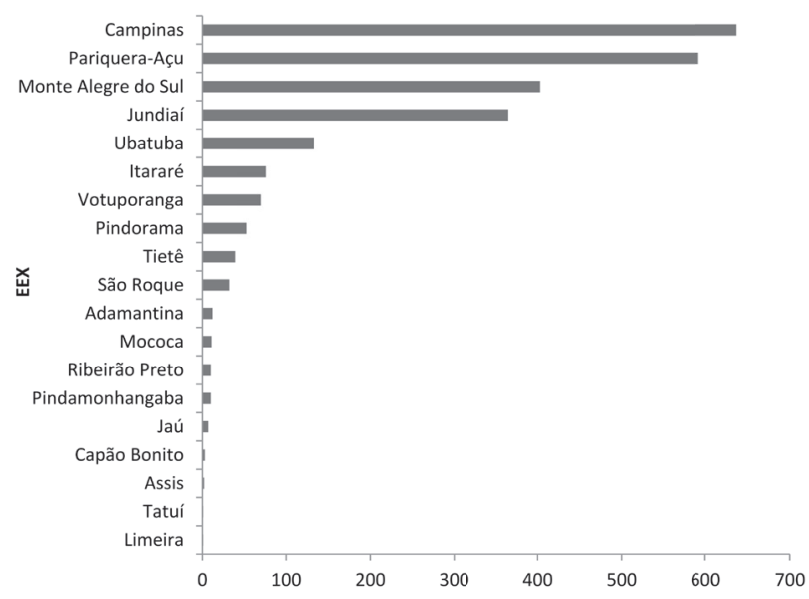

Figura 1 - Coletas de material botânico nas Estações Experimentais da Agência Paulista de Tecnologia dos Agronegócios (APTA)/ Instituto Agronômico de Campinas(IAC), Estado de São Paulo, Brasil. Número de coletas.

Figure 1 - Botanical collections in the Experimental Stations of Agência Paulista de Tecnologia dos Agronegócios (APTA)/ Instituto Agronômico de Campinas (IAC), São Paulo State, Brazil.

Number of collections. neste trabalho subestime a real diversidade dos fragmentos.

As famílias Fabaceae, Asteraceae, Rubiaceae, Myrtaceae e Poaceae foram as mais ricas, o que está de acordo com estudos florísticos e fitossociológicos realizados no Estado de São Paulo, como os de Silva \& Soares (2003), Yamamoto et al. (2005), Bernacci et al. (2006). É esperado que famílias como Asteraceae, Rubiaceae, Fabaceae e Poaceae sejam bastante amostradas em campo, pois esses táxons têm ampla distribuição geográfica, diferentes hábitos e grande número de espécies (Souza \& Lorenzi 2008, Judd et al. 2009). Além da riqueza, famílias como Fabaceae apresentam um grande número de espécies de valor econômico potencial (Martinelli et al. 2013), fato que foi constatado no presente estudo. Por outro lado, Bromeliaceae, que possui como um dos seus centros de diversidade o sudeste brasileiro (Benzing 1980), Cactaceae e Orchidaceae, esta última a família mais rica de Angiospermas (Souza \& Lorenzi 2008), foram subamostradas em decorrência da dificuldade de coleta, que muitas vezes exige a escalada das árvores, e de herborização dos materiais.

Embora as listagens de espécies ameaçadas estejam sujeitas a erros e lacunas, devido à falta de conhecimento sobre grande parte das espécies nativas, ainda assim indicam a necessidade de implementação de políticas públicas para a conservação dos táxons. De acordo com Martinelli \& Soares (2013), uma listagem considerada conservadora, foram encontradas duas espécies, uma vulnerável (Dalbergia nigra), na EEx de Monte Alegre do Sul, sob grande pressão de uso pelo valor de sua madeira, e outra em perigo de extinção (Nematanthus monanthos), por sua distribuição restrita, em Ubatuba. Dentre as 18 espécies consideradas ameaçadas segundo a Resolução SMA 48 (SMA 2004), Nectandra cissiflora, Myrcia flagellaris, Rudgea nobilis e Zanthoxylum petiolare, não são consideradas ameaçadas em Martinelli \& Soares (2013), mas os especialistas das famílias as recomendam como de interesse para a pesquisa e conservação. As espécies Gouania ulmifolia, Paspalum compressifolium, Peperomia nitida e Simira viridiflora, consideradas presumivelmente extintas, apresentam registros de coleta com menos de 50 anos. Aiouea acarodomatifera, Cissampelos pareira, Coussarea nodosa, Myracrodruon urundeuva, Ocotea nectandrifolia, Psychotria capitata, Piper xylosteoides e Trichilia hirta são espécies com coletas recentes ou distribuição geográfica ampla, características que 
Tabela 2. Famílias e espécies ocorrentes nas Estações Experimentais da Agência Paulista de Tecnologia dos Agronegócios (APTA)/Instituto Agronômico de Campinas(IAC), Estado de São Paulo, Brasil e exsicatas de referência do Herbário IAC. Adam: Adamantina; Assis: Ass; Camp: Campinas; Capão Bonito: CBon; Itararé: Itar; Jaú: Jaú; Jundiaí: Jund; Limeira: Lime; Moco: Mococa; MASu: Monte Alegre do Sul; PACU: Pariquera-Açu; Pinda: Pindamonhangaba; Pind: Pindorama; RPrt: Ribeirão Preto; SRoq: São Roque; Tatu: Tatuí; Tiet: Tietê; Ubat: Ubatuba; Votu: Votuporanga; *: espécie com potencial econômico de acordo com Walter et al. (2005a), Leite \& Coradin (2011) ou Aguiar et al. (2014); **: espécie ameaçada de extinção ou indicada para pesquisa e conservação de acordo com Martinelli \& Moraes (2013); ***: espécie ameaçada de extinção de acordo com Resolução SMA 48 (SMA 2004); \#: não ocorrência no Estado de SP; (ExIn): espécie exótica invasora.

Table 2. Families and species from the Experimental Stations of Agência Paulista de Tecnologia dos Agronegócios (APTA)/ Instituto Agronômico de Campinas (IAC), São Paulo State, Brazil and the reference voucher specimen in the IAC Herbarium. Adam: Adamantina; Assis: Ass; Camp: Campinas; Capão Bonito: CBon; Itararé: Itar; Jaú: Jaú; Jundiaí: Jund; Limeira: Lime; Moco: Mococa; MASu: Monte Alegre do Sul; PACU: Pariquera-Açu; Pinda: Pindamonhangaba; Pind: Pindorama; RPrt: Ribeirão Preto; SRoq: São Roque; Tatu: Tatuí; Tiet: Tietê; Ubat: Ubatuba; Votu: Votuporanga; *: species with economic potential according to Walter et al. (2005a), Leite \& Coradin (2011) or Aguiar et al. (2014); **: endangered species or suitable for research and conservation according to Martinelli \& Moraes (2013); ***: endangered species in accordance with Resolução SMA 48 (SMA 2004); \#: not occurring in the São Paulo State; (ExIn): exotic invasive species.

\begin{tabular}{|c|c|c|}
\hline Família / Espécie & Estação Experimental & Número IAC \\
\hline \multicolumn{3}{|l|}{ Acanthaceae } \\
\hline Aphelandra colorata (Vell.) Wassh. & Ubat & 4079 \\
\hline Aphelandra longiflora (Lindl.) Profice & SRoq, Tiet & 29161 \\
\hline Aphelandra ornata (Nees) T.Anderson & PAçu, Ubat & 35324 \\
\hline Aphelandra prismatica (Vell.) Hiern & Ubat & 4389 \\
\hline Hygrophila costata Nees & Ubat & 3527 \\
\hline Justicia cárnea Lindl. * & Camp, Ubat & 5339 \\
\hline Justicia lythroides (Nees) V.A.W.Graham * & Camp & 21845 \\
\hline Pseuderanthemum riedelianum (Nees) Radlk. & Camp & 21345 \\
\hline Ruellia brevifolia (Pohl) C.Ezcurra & Camp & 44577 \\
\hline Ruellia jussieuoides Schltdl. \& Cham. & Camp, MASu & 38006 \\
\hline Thunbergia alata Bojer ex Sims & Ubat & 2317 \\
\hline \multicolumn{3}{|l|}{ Adoxaceae } \\
\hline Sambucus canadensis L. * \# & MASu, Pind & 10698 \\
\hline \multicolumn{3}{|l|}{ Alstroemeriaceae } \\
\hline Bomarea edulis (Tussac) Herb. * & MASu & 7350 \\
\hline \multicolumn{3}{|l|}{ Amaranthaceae } \\
\hline Alternanthera dentata (Moench) Stuchlik ex R.E.Fr. * & Camp & 42931 \\
\hline Alternanthera philoxeroides (Mart.) Griseb. ${ }^{*}$ & Camp & 18032 \\
\hline Alternanthera pungens Kunth* & Pind & 4707 \\
\hline Alternanthera tenella Colla * & Camp, RPret & 23179 \\
\hline Amaranthus caudatus L. * & Camp & 9040 \\
\hline Amaranthus hybridus L. * & Camp & 19109 \\
\hline Amaranthus spinosus L. * & Camp, Pind & 19795 \\
\hline Amaranthus viridis L. * & Camp & 39565 \\
\hline Chamissoa altissima (Jacq.) Kunth & Camp & 47506 \\
\hline Gomphrena celosioides Mart. & Camp & 32126 \\
\hline Hebanthe eriantha (Poir.) Pedersen & Jund & 34667 \\
\hline Iresine diffusa Humb. \& Bonpl. ex Willd. * & MASu & 7362 \\
\hline \multicolumn{3}{|l|}{ Amaryllidaceae } \\
\hline Indeterminada & MASu & 37986 \\
\hline
\end{tabular}


Tabela 2 (continuação)

\begin{tabular}{l}
\hline Família / Espécie \\
\hline Anacardiaceae \\
Myracrodruon urundeuva Allemão \\
Schinus terebinthifolius Raddi * \\
Tapirira guianensis Aubl.
\end{tabular}

Anemiaceae

Anemia phyllitidis (L.) Sw.

Estação Experimental

Camp, Jund, RPret, SRoq,

Annonaceae

Annona dioica A.St.-Hil. *

Annona montana Macfad. *

Annona neosericea H.Rainer *

Annona sylvatica A.St.-Hil. *

Guatteria australis A.St.-Hil.

Unonopsis guatterioides (A.DC.) R.E.Fr.

Xylopia aromatica (Lam.) Mart.

Xylopia brasiliensis Spreng.

Apiaceae

Eryngium foetidum L. *

Eryngium pandanifolium Cham. \& Schltdl. *

Eryngium subinerme (H.Wolff) Mathias \& Constance *

Apocynaceae

Araujia sericifera Brot.

Asclepias curassavica L.

Aspidosperma parvifolium A.DC. *

Aspidosperma warmingii Müll.Arg. *

Condylocarpon sp.

Cryptostegia grandiflora R.Br.

Mandevilla hirsuta (A.Rich.) K.Schum.

Prestonia coalita (Vell.) Woodson

Prestonia dusenii (Malme) Woodson

Prestonia riedelii (Müll.Arg.) Markgr.

Rauvolfia sellowii Müll.Arg.

Tabernaemontana sp.

Tassadia sp.

Oxypetalum appendiculatum Mart.

Oxypetalum wightianum Hook. \& Arn.

Peplonia axillaris (Vell.) Fontella \& Rapini

Araceae

Anthurium pentaphyllum (Aubl.) G.Don *

Anthurium sellowianum Kunth *
Pind, Votu Ubat, MASu

Camp, PAçu, Votu

MASu, Pind

9227

Número IAC

47968

47521

47954

Votu

47955

Camp, PAçu, Pind

19893

PAçu

40399

Camp

46828

Ubat

47939

Pind

3428

Votu

48100

PAçu

47266

RPret

21432

MASu

7354

Itar

23009

Camp

9047

PAçu, Pind, Ubat

34092

$\mathrm{MASu}$

9066

PAçu

43573

PAçu

35761

Camp

9282

MASu

7357

Camp, MASu

32053

PAçu

29731

MASu, Camp

9225

MASu, Camp

14069

SRoq

28773

PAçu

35752

Camp, MASu, SRoq

30131

Camp

20167

PAçu

29098

PAçu

37248

PAçu, Ubat

33091 
Tabela 2 (continuação)

\begin{tabular}{l} 
Família / Espécie \\
\hline Monstera adansonii Schott * \\
Monstera praetermissa E.G.Gonç. \& Temponi * \\
Araliaceae
\end{tabular}

Dendropanax cuneatus (DC.) Decne. \& Planch.

Dendropanax monogynus (Vell.) Seem.

Oreopanax capitatus (Jacq.) Decne. \& Planch.

Arecaceae

Astrocaryum aculeatissimum (Schott) Burret

Syagrus sp. *

Aristolochiaceae

Aristolochia arcuata Mast.

Aristolochia gigantea Mart. \& Zucc.

Aristolochia labiata Willd.

Asteraceae

Acanthospermum australe (Loefl.) Kuntze

Achyrocline sp. *

Acmella brachyglossa Cass.

Adenostemma brasilianum (Pers.) Cass.

Ageratum sp. *

Ambrosia polystachya DC.

Aspilia sp. *

Baccharis anomala DC. *

Baccharis coridifolia DC. *

Baccharis crispa Spreng. *

Baccharis dentata (Vell.) G.M.Barroso *

Baccharis dracunculifolia DC. *

Baccharis montana DC. *

Baccharis rivularis Gardner*

Baccharis sagittalis (Less.) DC. *

Baccharis singularis (Vell.) G.M.Barroso *

Baccharis trinervis (Lam.) Pers. *

Bidens segetum Mart. ex Colla*

Calea pinnatifida (R.Br.) Less.

Calyptocarpus brasiliensis (Nees \& Mart.) B. Turner

Campuloclinium purpurascens (Sch.Bip. ex Baker) R.M.

King \& H.Rob.

Centratherum punctatum Cass.

Chaptalia sp.

Chromolaena congesta (Hook. \& Arn.) R.M.King \& H.Rob. *

Chromolaena laevigata (Lam.) R.M.King \& H.Rob. *

Chromolaena maximilianii (Schrad. ex DC.) R.M.King \& H.Rob. *

Conyza bonariensis (L.) Cronquist

Coreopsis sp.

\begin{tabular}{|c|c|}
\hline Estação Experimental & Número IA \\
\hline PAçu, Ubat & 36043 \\
\hline PAçu & 29794 \\
\hline $\begin{array}{l}\text { Jund, Moco, PAçu, MASu, } \\
\text { Votu }\end{array}$ & 47099 \\
\hline PAçu, Ubat & 37918 \\
\hline Ubat & 48073 \\
\hline PAçu & 37896 \\
\hline PAçu & 33097 \\
\hline Camp & 16661 \\
\hline Camp, Lime & 7678 \\
\hline Camp & 8052 \\
\hline Votu, Camp & 29568 \\
\hline PAçu, MASu, SRoq & 33065 \\
\hline Pinda, Ubat & 22434 \\
\hline Ubat & 3498 \\
\hline Camp & 36930 \\
\hline Camp & 813 \\
\hline Jund & 35541 \\
\hline PAçu & 35730 \\
\hline CBoni & 18202 \\
\hline Itar, MASu & 50430 \\
\hline Ubat & 5017 \\
\hline Camp, PAçu, RPret, Tiet & 47282 \\
\hline PAçu, Ubat & 29739 \\
\hline Votu & 29467 \\
\hline PAçu & 37880 \\
\hline PAçu, Ubat & 36000 \\
\hline Itar, Ubat & 49847 \\
\hline Camp & 9267 \\
\hline MASu & 7356 \\
\hline SRoq & 7289 \\
\hline Camp & 19959 \\
\hline Camp, PAçu, Ubat & 34146 \\
\hline Camp, Itar & 49699 \\
\hline Itar & 22798 \\
\hline Itar & 49728 \\
\hline Ubat & 3520 \\
\hline Camp, Ubat & 2405 \\
\hline Camp & 7618 \\
\hline
\end{tabular}


Tabela 2 (continuação)

\begin{tabular}{|c|c|c|}
\hline Família / Espécie & Estação Experimental & Número IAC \\
\hline Cosmos bipinnatus Cav. & Camp & 1584 \\
\hline Cyrtocymura scorpioides (Lam.) H.Rob. * & Camp, Jund, Ubat & 46971 \\
\hline Dasyanthina serrata (Less.) H.Rob. & Camp, Ubat & 20148 \\
\hline Dasyphyllum sp. * & Adam, MASu, Tiet, Votu & 30882 \\
\hline Delilia biflora (L.) Kuntze & Camp & 44573 \\
\hline Eclipta prostrata (L.) L. & Camp, RPret & 4572 \\
\hline Elephantopus mollis Kunth * & Jund, MASu & 28676 \\
\hline Emilia fosbergii Nicolson & Camp & 6895 \\
\hline Emilia sonchifolia (L.) DC. ex Wight & Camp & 4571 \\
\hline Erechtites valerianifolius (Wolf) DC. & Camp, Ubat & 2256 \\
\hline Fleischmannia remotifolia (DC.) R.M.King \& H.Rob. & Ubat & 4850 \\
\hline Galinsoga parviflora Cav. & Camp & 216 \\
\hline Gochnatia polymorpha (Less.) Cabrera* & Camp, Jund & 28829 \\
\hline Gochnatia velutina (Bong.) Cabrera * & Irar & 22782 \\
\hline Grazielia intermedia (DC.) R.M.King \& H.Rob. & MASu & 30068 \\
\hline Grindelia sp. & Camp & 7330 \\
\hline Heterocondylus alatus (Vell.) R.M.King \& H.Rob. & Ubat & 4845 \\
\hline Hieracium sp. & Itar & 50228 \\
\hline Hypochaeris chillensis (Kunth) Britton & Camp & 25126 \\
\hline Jaegeria hirta (Lag.) Less. & Ubat & 3010 \\
\hline Jungia floribunda Less. & Camp, SRoq & 28747 \\
\hline Lessingianthus glabratus (Less.) H.Rob. & Camp & 20345 \\
\hline Lessingianthus macrophyllus (Less.) H.Rob. & MASu, Ubat & 14065 \\
\hline Melampodium divaricatum (Rich. ex Pers.) DC.\# & Camp & 20860 \\
\hline Mikania chlorolepis Baker * & Ubat & 5643 \\
\hline Mikania cordifolia (L.f.) Willd. * & Camp & 20183 \\
\hline Mikania glomerata Spreng. * & Ubat & 4843 \\
\hline Mikania hirsutissima DC. * & Itar & 49743 \\
\hline Mikania trinervis Hook. \& Arn. * & Ubat & 4829 \\
\hline Mutisia sp. & Camp, PAçu & 47283 \\
\hline Orthopappus angustifolius (Sw.) Gleason & Camp & 20412 \\
\hline Parthenium argentatum A.Gray & Camp & 9299 \\
\hline Piptocarpha axillaris (Less.) Baker * & PAçu & 35744 \\
\hline Piptocarpha notata (Less.) Baker* & PAçu & 20313 \\
\hline Piptocarpha oblonga (Gardner) Baker * & Ubat & 4839 \\
\hline Porophyllum ruderale (Jacq.) Cass. & Camp, MASu, SRoq & 19648 \\
\hline Praxelis pauciflora (Kunth) R.M.King \& H.Rob. \# & Camp & 25263 \\
\hline Schkuhria pinnata (Lam.) Kuntze & Camp & 19151 \\
\hline Senecio brasiliensis (Spreng.) Less. * & Camp & 10319 \\
\hline Sigesbeckia orientalis L. & Camp & 20816 \\
\hline Sonchus asper (L.) Hill & Camp & 7314 \\
\hline Sonchus oleraceus L. & Camp & 6669 \\
\hline Sphagneticola trilobata (L.) Pruski & Camp & 25227 \\
\hline
\end{tabular}


Tabela 2 (continuação)

\begin{tabular}{|c|c|c|}
\hline Família / Espécie & Estação Experimental & Número IAC \\
\hline Stevia rebaudiana (Bertoni) Bertoni *,\# & Itar & 22799 \\
\hline Steyermarkina pyrifolia (DC.) R.M.King \& H.Rob. & Ubat & 4836 \\
\hline Stifftia parviflora (Leandro) D.Don & Ubat & 4830 \\
\hline Symphyopappus compressus (Gardner) B.L.Rob. & PAçu & 29718 \\
\hline Symphyopappus itatiayensis (Hieron.) R.M.King \& H.Rob. & Ubat & 5337 \\
\hline Tagetes minuta L. * & MASu & 29004 \\
\hline Tilesia baccata (L.f.) Pruski & Camp, Pind & 19102 \\
\hline Trichocline sp. * & Camp & 854 \\
\hline Trichogoniopsis adenantha (DC.) R.M.King \& H.Rob. & Jund & 28658 \\
\hline Trixis antimenorrhoea (Schrank) Kuntze & Pind, Ubat & 4822 \\
\hline Vernonanthura discolor (Spreng.) H.Rob. * & Ubat & 5316 \\
\hline Vernonanthura lindbergii (Baker) H.Rob. * & Ubat & 5640 \\
\hline Vernonanthura phosphorica (Vell.) H.Rob. * & Ubat & 4821 \\
\hline Wedelia sp. & Camp, MASu & 37868 \\
\hline \multicolumn{3}{|l|}{ Balanophoraceae } \\
\hline Scybalium fungiforme Schott \& Endl. & MASu & 28963 \\
\hline \multicolumn{3}{|l|}{ Basellaceae } \\
\hline Basella alba $\mathrm{L}$. & Camp & 18773 \\
\hline \multicolumn{3}{|l|}{ Begoniaceae } \\
\hline Begonia maculata (Raddi) Herb. * & MASu & 9221 \\
\hline \multicolumn{3}{|l|}{ Bignoniaceae } \\
\hline Adenocalymma bracteatum (Cham.) DC. & Camp, Tiet & 16683 \\
\hline Adenocalymma dusenii Kraenzl. \# & PAçu & 35470 \\
\hline Cuspidaria floribunda (DC.) A.H.Gentry & Pind & 4722 \\
\hline Cybistax antisyphilitica (Mart.) Mart. & MASu & 9078 \\
\hline Dolichandra unguis-cati (L.) L.G.Lohmann & Jund & 17321 \\
\hline Fridericia florida (DC.) L.G.Lohmann & Camp, Pind, RPret & 4736 \\
\hline Fridericia speciosa Mart. & Camp, Pind & 859 \\
\hline Handroanthus impetiginosus Mattos * & Pind & 2144 \\
\hline Handroanthus vellosoi (Toledo) Mattos * & MASu & 7999 \\
\hline Jacaranda caroba (Vell.) DC. * & Camp & 24180 \\
\hline Lundia cordata (Vell.) DC. & Ubat & 4864 \\
\hline Mansoa difficilis (Cham.) Bureau \& K.Schum. & Camp & 32209 \\
\hline Pyrostegia venusta (Ker Gawl.) Miers * & MASu, Tiet & 37979 \\
\hline Stizophyllum perforatum (Cham.) Miers & Pind & 4688 \\
\hline Tynanthus sp. & Jund & 37371 \\
\hline Zeyheria tuberculosa (Vell.) Bureau ex Verl. & MASu & 8298 \\
\hline \multicolumn{3}{|l|}{ Bixaceae } \\
\hline Bixa orellana L. * & $\mathrm{MASu}$ & 9077 \\
\hline \multicolumn{3}{|l|}{ Blechnaceae } \\
\hline Blechnum schomburgkii (Klotzsch) C.Chr. & Itar & 49835 \\
\hline \multicolumn{3}{|l|}{ Boraginaceae } \\
\hline Cordia americana (L.) Gottschling \& J.S.Mill. * & Camp, Pind & 29915 \\
\hline Cordia anabaptista Cham. * & RPret & 3871 \\
\hline
\end{tabular}


Tabela 2 (continuação)

\begin{tabular}{|c|c|c|}
\hline Família / Espécie & Estação Experimental & Número IAC \\
\hline Cordia ecalyculata Vell. * & Jund, MASu & 8010 \\
\hline Cordia sellowiana Cham. * & Camp, MASu & 33010 \\
\hline Cordia taguahyensis Vell. * & Ubat & 46848 \\
\hline Cordia trichotoma (Vell.) Arráb. ex Steud. * & Camp, MASu & 36941 \\
\hline Heliotropium transalpinum Vell. & Pind & 4717 \\
\hline Tournefortia paniculata Cham. & Camp, Pind & 20176 \\
\hline \multicolumn{3}{|l|}{ Brassicaceae } \\
\hline Coronopus didymus (L.) Sm. & Camp & 6452 \\
\hline \multicolumn{3}{|l|}{ Bromeliaceae } \\
\hline Aechmea nudicaulis (L.) Griseb. * & PAçu & 35320 \\
\hline Aechmea organensis Wawra * & PAçu & 33087 \\
\hline Billbergia amoena (Lodd.) Lindl. * & PAçu & 28892 \\
\hline Billbergia distachia (Vell.) Mez * & $\mathrm{MASu}$ & 37977 \\
\hline Nidularium procerum Lindm. $*$ & PAçu & 28860 \\
\hline Pseudananas sagenarius (Arruda) Camargo * & Adam & 30878 \\
\hline Tillandsia pohliana $\mathrm{Mez} *$ & Camp, Tiet & 35350 \\
\hline Tillandsia recurvata (L.) L. * & SRoq, Tiet & 28792 \\
\hline Tillandsia stricta Sol. * & Jund, PAçu & 37899 \\
\hline Tillandsia tricholepis Baker* & Camp, Tiet & 32171 \\
\hline Vriesea carinata Wawra * & PAçu & 28898 \\
\hline Vriesea ensiformis (Vell.) Beer* & PAçu & 28900 \\
\hline Vriesea philippocoburgii Wawra * & PAçu & 33103 \\
\hline Vriesea procera (Mart. ex Schult. \& Schult.f.) Wittm. * & PAçu & 36024 \\
\hline \multicolumn{3}{|l|}{ Cactaceae } \\
\hline Epiphyllum phyllanthus (L.) Haw. & $\mathrm{MASu}$ & 30208 \\
\hline Lepismium cruciforme (Vell.) Miq. & Tiet & 29189 \\
\hline Lepismium warmingianum (K.Schum.) Barthlott & Tiet & 29188 \\
\hline Rhipsalis pachyptera Pfeiff. & PAçu & 33086 \\
\hline \multicolumn{3}{|l|}{ Campanulaceae } \\
\hline Lobelia camporum Pohl & Itar & 49758 \\
\hline Siphocampylus macropodus (Thunb.) G.Don & MASu & 7368 \\
\hline \multicolumn{3}{|l|}{ Cannabaceae } \\
\hline Celtis fluminensis Carauta & Camp & 53531 \\
\hline Celtis pubescens (Kunth) Spreng. & Camp & 46799 \\
\hline Celtis spinosa Spreng. & Camp & 49438 \\
\hline Trema micrantha (L.) Blume * & $\begin{array}{c}\text { Camp, MASu, PAçu, Pind, } \\
\text { SRoq }\end{array}$ & 49469 \\
\hline \multicolumn{3}{|l|}{ Cardiopteridaceae } \\
\hline Citronella gongonha (Mart.) R.A.Howard & Camp & 31961 \\
\hline Citronella megaphylla (Miers) R.A.Howard & Camp & 41693 \\
\hline Citronella paniculata (Mart.) R.A.Howard & Camp, MASu & 18772 \\
\hline \multicolumn{3}{|l|}{ Caryophyllaceae } \\
\hline Drymaria cordata (L.) Willd. ex Roem. \& Schult. & Camp & 21847 \\
\hline
\end{tabular}


Tabela 2 (continuação)

Família / Espécie

Estação Experimental

Número IAC

Celastraceae

Hippocratea volubilis L.

Jund

35535

Maytenus aquifolia Mart. *

Camp, Jund

32064

Maytenus floribunda Reissek *

Votu

30501

Maytenus salicifolia Reissek *

Jund

33689

Chrysobalanaceae

Hirtella hebeclada Moric. ex DC. *

Camp, PAçu

33033

Licania kunthiana Hook.f.

PAçu

32270

Parinari excelsa Sabine

PAçu

31985

Clusiaceae

Clusia lanceolata Cambess. *

Ubat

48306

Tovomitopsis sp.

MASu

9088

Commelinaceae

Dichorisandra incurva Mart.

Dichorisandra paranaensis D.Maia, Cervi \& Tardivo

Jund, MASu, PAçu

37724

Dichorisandra pubescens Mart. ex Schult \& Schult.f.

PAçu

33077

PAçu

29777

Tradescantia sp.

Camp, PAçu

37869

Tripogandra diuretica (Mart.) Handlos

Jund, MASu, PAçu

32975

Connaraceae

Connarus regnellii G.Schellenb.

Connarus rostratus (Vell.) L.B.Sm.

Camp, Jund, MASu

32207

PAçu

35525

Convolvulaceae

Ipomoea cairica (L.) Sweet *

Ipomoea hederifolia $\mathrm{L}$. *

Ipomoea quamoclit L. *

Camp, PAçu

47676

Camp, Moco, RPret

19146

PAçu

36672

Costaceae

Costus sp.

PAçu

35745

Cucurbitaceae

Fevillea passiflora Vell. *

Momordica charantia L. *

Sicana odorifera (Vell.) Naudin *

MASu, Ubat

49548

Camp

19124

Votu

29307

Cunoniaceae

Lamanonia ternata Vell.

Pind

4645

Cyclanthaceae

Cyclanthus sp.

PAçu

36028

Cyperaceae

Cyperus luzulae (L.) Retz.

Pind

4675

Cyperus odoratus $\mathrm{L}$.

Pinda

9356

Cyperus prolixus Kunth

MASu

7352

Cyperus rotundus $\mathrm{L}$.

Camp, Ubat

742

Eleocharis montana (Kunth) Roem. \& Schult.

Pinda

9354

Fuirena umbellata Rottb.

Pinda

9357

Fuirena robusta Kunth

Camp, PAçu

19666 
Tabela 2 (continuação)

\begin{tabular}{|c|c|c|}
\hline Família / Espécie & Estação Experimental & Número IAC \\
\hline Pycreus lanceolatus (Poir.) C.B.Clarke & Pinda & 9355 \\
\hline Rhynchospora corymbosa (L.) Britton & Pind & 4673 \\
\hline Rhynchospora exaltata Kunth & Camp & 20174 \\
\hline Scleria gaertneri Raddi \# & MASu, PAçu & 30087 \\
\hline Scleria latifolia $\mathrm{Sw}$. & PAçu, Ubat & 35750 \\
\hline Scleria plusiophylla Steud. & Jund, MASu, PAçu & 35287 \\
\hline Scleria secans (L.) Urb. & PAçu & 35770 \\
\hline \multicolumn{3}{|l|}{ Dilleniaceae } \\
\hline Davilla rugosa Poir. * & PAçu & 35373 \\
\hline Tetracera sp. & PAçu & 37927 \\
\hline \multicolumn{3}{|l|}{ Dioscoreaceae } \\
\hline Dioscorea alata $\mathrm{L} . *$ & Camp & 16679 \\
\hline Dioscorea bulbifera L. * & Camp & 6247 \\
\hline Dioscorea hassleriana Chodat* & Ubat & 30783 \\
\hline Dioscorea multiflora Mart. ex Griseb. * & MASu & 30195 \\
\hline Dioscorea stegelmanniana R.Knuth * & Ubat & 5634 \\
\hline Dioscorea trifida L.f. * & Camp & 6265 \\
\hline \multicolumn{3}{|l|}{ Dryopteridaceae } \\
\hline Dryopteris sp. & Ubat & 2117 \\
\hline \multicolumn{3}{|l|}{ Ebenaceae } \\
\hline Diospyros inconstans Jacq. * & Jund, MASu & 30241 \\
\hline \multicolumn{3}{|l|}{ Elaeocarpaceae } \\
\hline Sloanea hirsuta (Schott) Planch. ex Benth. * & Camp, PAçu, Ubat & 49483 \\
\hline \multicolumn{3}{|l|}{ Ericaceae } \\
\hline Agarista pulchella Cham. ex G.Don & Itar & 22775 \\
\hline Gaylussacia brasiliensis (Spreng.) Meisn. & Itar, PAçu & 22774 \\
\hline \multicolumn{3}{|l|}{ Eriocaulaceae } \\
\hline Paepalanthus albo-vaginatus Silveira \# & Itar & 49844 \\
\hline Paepalanthus elongatus (Bong.) Körn. \# & Itar & 49846 \\
\hline Paepalanthus tessmannii Moldenke & Itar & 49843 \\
\hline Syngonanthus nitens Ruhland & Itar & 49845 \\
\hline \multicolumn{3}{|l|}{ Erythroxylaceae } \\
\hline Erythroxylum cuneifolium (Mart.) O.E.Schulz * & Votu & 29476 \\
\hline Erythroxylum microphyllum A.St.-Hil. * & Itar & 49812 \\
\hline Erythroxylum suberosum A.St.-Hil. * & Camp & 53529 \\
\hline \multicolumn{3}{|l|}{ Escalloniaceae } \\
\hline Escallonia farinacea A.St.-Hil. * & Itar & 50221 \\
\hline \multicolumn{3}{|l|}{ Euphorbiaceae } \\
\hline Acalypha villosa Jacq. & Pind & 3432 \\
\hline Actinostemon concepcionis (Chodat \& Hassl.) Hochr. & Adam, Jund & 47985 \\
\hline Actinostemon klotzschii (Didr.) Pax & Jund, Pind & 29335 \\
\hline Alchornea glandulosa Poepp. \& Endl. * & Camp, MASu, PAçu & 34790 \\
\hline Alchornea sidifolia Müll.Arg. * & Camp & 53530 \\
\hline
\end{tabular}


Tabela 2 (continuação)

\begin{tabular}{|c|c|c|}
\hline Família / Espécie & Estação Experimental & Número IAC \\
\hline Alchornea triplinervia (Spreng.) Müll.Arg. * & PAçu & 35723 \\
\hline Aparisthmium cordatum (A.Juss.) Baill. & PAçu & 35782 \\
\hline Astraea lobata (L.) Klotzsch & Camp & 45320 \\
\hline Croton celtidifolius Baill. * & MASu & 37984 \\
\hline Croton floribundus Spreng. * & $\begin{array}{l}\text { Camp, Jund, MASu, Pind, } \\
\text { Ubat }\end{array}$ & 46976 \\
\hline Croton glandulosus L. * & Camp, Pind & 23170 \\
\hline Croton lundianus (Didr.) Müll.Arg. * & Camp & 36932 \\
\hline Croton piptocalyx Müll.Arg. * & Camp & 49488 \\
\hline Croton priscus Croizat* & Camp & 46970 \\
\hline Croton rottlerifolius Baill. * & Camp & 46974 \\
\hline Croton sclerocalyx (Didr.) Müll.Arg. * & RPret, SRoq & 3972 \\
\hline Croton triqueter Lam. * & PAçu, Votu & 36007 \\
\hline Croton urucurana Baill. * & Moco, MASu, Pind & 47517 \\
\hline Dalechampia pentaphylla Lam. & Camp & 45325 \\
\hline Dalechampia stipulacea Müll.Arg. & Pind & 4690 \\
\hline Euphorbia comosa Vell. & Camp & 6263 \\
\hline Euphorbia heterophylla $\mathrm{L}$. & Camp,Pind & 25291 \\
\hline Euphorbia hirta L. & Camp,Pind & 4551 \\
\hline Jatropha curcas L. * & Camp,Pind & 25512 \\
\hline Jatropha gossypiifolia L. * & Camp & 5139 \\
\hline Manihot carthaginensis (Jacq.) Müll.Arg. *,\# & Camp & 6741 \\
\hline Manihot pilosa Pohl * & Camp & 19034 \\
\hline Maprounea guianensis Aubl. & PAçu & 35368 \\
\hline Microstachys hispida (Mart.) Govaerts & Votu & 29557 \\
\hline Pausandra morisiana (Casar.) Radlk. & PAçu & 37915 \\
\hline Pera glabrata (Schott) Poepp. ex Baill. & Camp, PAçu & 35352 \\
\hline Sapium glandulosum (L.) Morong * & PAçu, Votu & 36696 \\
\hline Sebastiania brasiliensis Spreng. * & Camp & 53540 \\
\hline Tragia alienata (Didr.) Múlgura \& M.M.Gut. & MASu & 48106 \\
\hline Tragia volubilis $\mathrm{L}$. & Pind & 4744 \\
\hline \multicolumn{3}{|l|}{ Fabaceae } \\
\hline Abarema brachystachya (DC.) Barneby \& J.W.Grimes & Camp, Ubat & 31967 \\
\hline Abarema langsdorffii (Benth.) Barneby \& J.W.Grimes & PAçu & 19681 \\
\hline Acacia plumosa Lowe \# & Pind, RPret, Ubat & 6180 \\
\hline Aeschynomene sp. * & Jund & 30240 \\
\hline Albizia sp. * & Camp, PAçu & 7277 \\
\hline Anadenanthera colubrina (Vell.) Brenan * & MASu & 8308 \\
\hline Anadenanthera colubrina var. cebil (Griseb.) Altschul * & Pind & 2148 \\
\hline Anadenanthera peregrina (L.) Speg. * & RPret & 19457 \\
\hline Andira fraxinifolia Benth. * & Ubat & 23463 \\
\hline Bauhinia forficata Link * & Camp, MASu & 32021 \\
\hline Bauhinia longifolia (Bong.) Steud. * & Camp, Jund, MASu & 32999 \\
\hline Bauhinia pentandra (Bong.) Vogel ex Steud. * & Votu & 48255 \\
\hline
\end{tabular}


Tabela 2 (continuação)

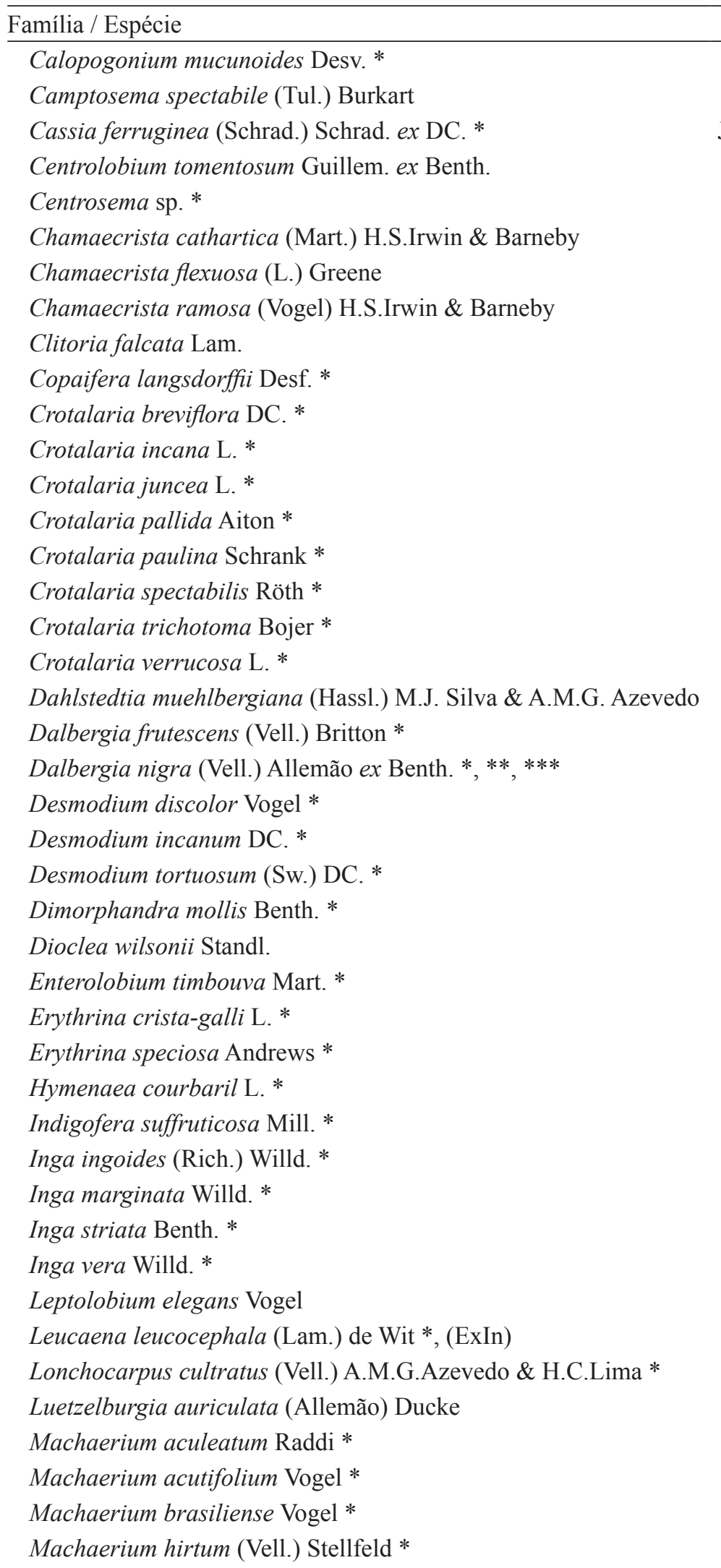

\begin{tabular}{|c|c|}
\hline Estação Experimental & Número IAC \\
\hline Camp & 6765 \\
\hline MASu, Tiet & 7149 \\
\hline Jund, MASu, Pind, Ubat & 37459 \\
\hline MASu & 8315 \\
\hline Votu & 30486 \\
\hline Camp & 4156 \\
\hline Camp & 37211 \\
\hline Itar & 22796 \\
\hline Camp, MASu & 30080 \\
\hline MASu & 28979 \\
\hline Jund, SRoq & 30262 \\
\hline MASu, RPret & 44158 \\
\hline Camp & 6718 \\
\hline Camp, PAçu & 33047 \\
\hline Camp, MASu & 28958 \\
\hline Camp & 6402 \\
\hline Camp & 6775 \\
\hline Camp & 7190 \\
\hline MASu, SRoq & 16270 \\
\hline PAçu, Ubat & 28847 \\
\hline MASu & 19486 \\
\hline Camp, MASu & 19135 \\
\hline Camp, Pind & 3418 \\
\hline SRoq & 28798 \\
\hline Votu, Ass & 29533 \\
\hline PAçu & 37877 \\
\hline Pind & 2133 \\
\hline Camp, MASu & 8312 \\
\hline Camp, PAçu & 36694 \\
\hline MASu, PAçu, Pind & 28999 \\
\hline Camp, MASu, Ubat & 20350 \\
\hline Camp, Pind, RPret & 8988 \\
\hline Jaú, Pind, SRoq, Ubat & 7631 \\
\hline Jaú, MASu & 7639 \\
\hline Camp, MASu & 6646 \\
\hline Votu & 29495 \\
\hline Camp, Pind & 4129 \\
\hline Camp, MASu & 49468 \\
\hline MASu & 7994 \\
\hline MASu & 9219 \\
\hline Votu & 29448 \\
\hline Jund, Pind & 21305 \\
\hline Camp,Votu & 19846 \\
\hline
\end{tabular}


Tabela 2 (continuação)

\begin{tabular}{|c|c|c|}
\hline Família / Espécie & Estação Experimental & Número IAC \\
\hline Machaerium nyctitans (Vell.) Benth. * & MASu, PAçu, Tiet & 7991 \\
\hline Machaerium scleroxylon Tul. * & MASu & 8000 \\
\hline Machaerium stipitatum (DC.) Vogel * & MASu, SRoq, Tiet & 8296 \\
\hline Machaerium vestitum Vogel * & $\mathrm{MASu}$ & 42773 \\
\hline Machaerium villosum Vogel * & MASu, Pind & 9069 \\
\hline Macroptilium atropurpureum (Sessé \& Moc. ex DC.) Urb. * & Votu & 30526 \\
\hline Macroptilium bracteatum (Nees \& Mart.) Maréchal \& Baudet* & Moco & 23115 \\
\hline Macroptilium lathyroides(L.) Urb. * & Camp & 19777 \\
\hline Macroptilium panduratum (Mart. ex Benth.) Maréchal \& Baudet * & Camp, MASu & 9220 \\
\hline Mimosa diplotricha C.Wright ex Sauvalle * & MASu & 30158 \\
\hline Mimosa invisa Mart. ex Colla * & Ubat & 30781 \\
\hline Mimosa pudica L. * & PAçu, Ubat & 33119 \\
\hline Mimosa velloziana Mart. * & MASu & 14068 \\
\hline Mucuna urens (L.) Medik. & PAçu & 29798 \\
\hline Myroxylon peruiferum L.f. * & Moco, Pind & 47516 \\
\hline Ormosia arborea (Vell.) Harms & $\mathrm{MASu}$ & 9086 \\
\hline Peltophorum dubium (Spreng.) Taub. * & Camp, MASu & 10702 \\
\hline Piptadenia gonoacantha (Mart.) J.F.Macbr. * & Jund, MASu, Pind & 32982 \\
\hline Platycyamus regnellii Benth. & Camp & 7290 \\
\hline Platypodium elegans Vogel & Camp, Pind & 29927 \\
\hline Poincianella pluviosa (DC.) L.P.Queiroz \# & MASu & 8014 \\
\hline Pseudopiptadenia warmingii (Benth.) G.P.Lewis \& M.P.Lima & PAçu & 35442 \\
\hline Rhynchosia edulis Griseb. * & SRoq & 28768 \\
\hline Senegalia grandistipula (Benth.) Seigler \& Ebinger * & PAçu & 35421 \\
\hline Senegalia lacerans (Benth.) Seigler \& Ebinger * & Ubat & 5340 \\
\hline Senegalia martii (Benth.) Seigler \& Ebinger * & Camp & 898 \\
\hline Senegalia polyphylla (DC.) Britton \& Rose * & Camp, MASu, Pind & 8297 \\
\hline Senegalia tenuifolia $($ L.) Britton \& Rose * & Tiet & 29155 \\
\hline Senna alata (L.) Roxb. * & Camp & 7294 \\
\hline Senna cernua (Balb.) H.S.Irwin \& Barneby * & Camp & 7292 \\
\hline Senna macranthera (DC. ex Collad.) H.S.Irwin \& Barneby * & Camp & 11247 \\
\hline Senna multijuga (Rich.) H.S.Irwin \& Barneby * & MASu, PAçu, Ubat & 30211 \\
\hline Senna obtusifolia (L.) H.S.Irwin \& Barneby * & Camp, Pinda & 55 \\
\hline Senna occidentalis (L.) Link * & Pind & 3444 \\
\hline Senna siamea (Lam.) H.S.Irwin \& Barneby * & Camp & 7280 \\
\hline Senna silvestris (Vell.) H.S.Irwin \& Barneby * & Camp, PAçu & 19022 \\
\hline Senna spectabilis (DC.) H.S.Irwin \& Barneby * & Camp & 18173 \\
\hline Senna splendida (Vogel) H.S.Irwin \& Barneby * & Camp, Jund & 5597 \\
\hline Senna tropica (Vell.) H.S.Irwin \& Barneby * & Ubat & 5321 \\
\hline Swartzia langsdorffii Raddi & PAçu & 19069 \\
\hline Swartzia oblata R.S.Cowan & Ubat & 5635 \\
\hline Tephrosia candida DC. & Jund, Pind & 36326 \\
\hline Teramnus uncinatus (L.) Sw. & Moco & 16253 \\
\hline
\end{tabular}


Tabela 2 (continuação)

\begin{tabular}{|c|c|c|}
\hline Família / Espécie & Estação Experimental & Número IAC \\
\hline Zornia glabra Desv. * & PAçu & 29741 \\
\hline Zornia reticulata $\mathrm{Sm} . *$ & Camp & 20965 \\
\hline \multicolumn{3}{|l|}{ Gesneriaceae } \\
\hline Besleria longimucronata Hoehne & Ubat & 4859 \\
\hline Codonanthe devosiana Lem. & Ubat & 30776 \\
\hline Codonanthe gracilis (Mart.) Hanst. & PAçu, Ubat & 35319 \\
\hline Helia oblongifolia Mart. & Itar & 49816 \\
\hline Nematanthus fissus (Vell.) L.E.Skog & Ubat & 4856 \\
\hline Nematanthus monanthos (Vell.) Chautems **, *** & Ubat & 5778 \\
\hline Sinningia allagophylla (Mart.) Wiehler * & Camp & 5127 \\
\hline Sinningia elatior (Kunth) Chautems * & Camp, Itar & 18447 \\
\hline Sinningia speciosa (Lodd.) Hiern * & Camp & 7629 \\
\hline \multicolumn{3}{|l|}{ Heliconiaceae } \\
\hline Heliconia sp. * & MASu, Pind & 38008 \\
\hline \multicolumn{3}{|l|}{ Iridaceae } \\
\hline Sisyrinchium restioides Spreng. & Itar & 49823 \\
\hline Sisyrinchium vaginatum Spreng. & Itar & 49825 \\
\hline \multicolumn{3}{|l|}{ Juncaceae } \\
\hline Juncus sp. & Itar & 50222 \\
\hline \multicolumn{3}{|l|}{ Lacistemataceae } \\
\hline Lacistema hasslerianum Chodat & Adam, Camp & 30871 \\
\hline \multicolumn{3}{|l|}{ Lamiaceae } \\
\hline Aegiphila integrifolia (Jacq.) Moldenke & Camp & 35326 \\
\hline Hyptis brevipes Poit. * & Camp & 36928 \\
\hline Hyptis spicigera Lam. * & Camp & 19913 \\
\hline Ocimum sp. * & Camp & 46972 \\
\hline Rhabdocaulon lavanduloides (Benth.) Epling & Itar & 50224 \\
\hline Salvia sp. & Camp & 32998 \\
\hline Vitex polygama Cham. * & MASu & 14075 \\
\hline \multicolumn{3}{|l|}{ Lauraceae } \\
\hline Aiouea acarodomatifera Kosterm. *** & PAçu & 47280 \\
\hline Aniba viridis $\mathrm{Mez} *$ & PAçu, Ubat & 47273 \\
\hline Cassytha filiformis L. & Itar, Votu & 49807 \\
\hline Cinnamomum triplinerve (Ruiz \& Pav.) Kosterm. & PAçu & 37221 \\
\hline Cryptocarya aschersoniana $\mathrm{Mez}$ & Camp & 48104 \\
\hline Cryptocarya mandioccana Meisn. & PAçu & 35516 \\
\hline Cryptocarya moschata Nees \& Mart. & SRoq & 30336 \\
\hline Endlicheria paniculata (Spreng.) J.F.Macbr. & $\begin{array}{l}\text { Camp, MASu, PAçu, Pind, } \\
\text { Ubat }\end{array}$ & 46801 \\
\hline Licaria armeniaca (Nees) Kosterm. & Ubat & 48316 \\
\hline Nectandra cissiflora Nees $*, * *, * * *$ & Votu & 47970 \\
\hline Nectandra grandiflora Nees * & Camp, MASu & 47089 \\
\hline Nectandra lanceolata Nees * & MASu & 14073 \\
\hline Nectandra megapotamica (Spreng.) Mez * & $\begin{array}{c}\text { Adam, Camp, MASu, } \\
\text { Pind, RPret }\end{array}$ & 47986 \\
\hline
\end{tabular}


Tabela 2 (continuação)

\begin{tabular}{|c|c|c|}
\hline Família / Espécie & Estação Experimental & Número IAC \\
\hline Nectandra membranacea (Sw.) Griseb. * & Ubat & 48296 \\
\hline Nectandra nitidula Nees * & Camp & 35345 \\
\hline Nectandra oppositifolia Nees * & PAçu, Ubat & 35994 \\
\hline Nectandra reticulata (Ruiz \& Pav.) Mez * & Ubat & 19960 \\
\hline Ocotea brachybotrya (Meisn.) Mez * & Ubat & 49541 \\
\hline Ocotea catharinensis $\mathrm{Mez} *$ & Jund & 35531 \\
\hline Ocotea corymbosa (Meisn.) Mez * & Pind & 4684 \\
\hline Ocotea daphnifolia (Meisn.) Mez * & PAçu & 43578 \\
\hline Ocotea diospyrifolia (Meisn.) Mez * & MASu & 49422 \\
\hline Ocotea dispersa (Nees \& Mart.) Mez * & PAçu, Ubat & 35362 \\
\hline Ocotea minarum (Nees \& Mart.) Mez * & Votu & 30561 \\
\hline Ocotea nectandrifolia $\mathrm{Mez} *, * * *$ & PAçu & 35363 \\
\hline Ocotea odorifera (Vell.) Rohwer * & Jund, MASu, PAçu & 35277 \\
\hline Ocotea puberula (Rich.) Nees * & MASu, Tiet, Ubat & 29032 \\
\hline Ocotea velloziana (Meisn.) Mez * & Camp & 53524 \\
\hline \multicolumn{3}{|l|}{ Lecythidaceae } \\
\hline Cariniana estrellensis (Raddi) Kuntze * & Camp, MASu & 33008 \\
\hline Cariniana legalis (Mart.) Kuntze * & $\mathrm{MASu}$ & 8004 \\
\hline \multicolumn{3}{|l|}{ Loganiaceae } \\
\hline Spigelia sp. & PAçu & 33061 \\
\hline Strychnos brasiliensis Mart. & MASu & 38001 \\
\hline \multicolumn{3}{|l|}{ Loranthaceae } \\
\hline Struthanthus concinnus Mart. & PAçu & 53017 \\
\hline Struthanthus flexicaulis Mart. & MASu & 7366 \\
\hline \multicolumn{3}{|l|}{ Lythraceae } \\
\hline Cuphea calophylla Cham. \& Schltdl. * & Itar & 22777 \\
\hline Cuphea carthagenensis (Jacq.) J.Macbr. * & Ubat & 3507 \\
\hline Lafoensia sp. * & MASu & 9064 \\
\hline \multicolumn{3}{|l|}{ Malpighiaceae } \\
\hline Bunchosia maritima (Vell.) J.F.Macbr. & Camp & 47528 \\
\hline Byrsonima guilleminiana A.Juss. * & Votu & 48105 \\
\hline Carolus chlorocarpus (A.Juss.) W.R.Anderson & Pind & 6177 \\
\hline Heteropterys intermedia (A.Juss.) Griseb. & MASu, Ubat & 9231 \\
\hline Stigmaphyllon affine A.Juss. & Camp & 413 \\
\hline Stigmaphyllon puberulum Griseb. & Ubat & 4844 \\
\hline \multicolumn{3}{|l|}{ Malvaceae } \\
\hline Abutilon fluviatile (Vell.) K.Schum. & Camp & 44575 \\
\hline Abutilon longifolium K.Schum. & MASu & 37983 \\
\hline Abutilon venosum K.Schum. & MASu & 29010 \\
\hline Byttneria catalpifolia Jacq. & Pind & 6176 \\
\hline Ceiba pubiflora (A.St.-Hil.) K.Schum. * & Camp & 8170 \\
\hline Ceiba speciosa (A.St.-Hil.) Ravenna * & Camp, MASu & 19131 \\
\hline Eriotheca candolleana (K.Schum.) A.Robyns & MASu & 8002 \\
\hline
\end{tabular}


Tabela 2 (continuação)

\begin{tabular}{|c|c|c|}
\hline Família / Espécie & Estação Experimental & Número IAC \\
\hline Guazuma ulmifolia Lam. & Camp, Moco, Votu & 34796 \\
\hline Helicteres brevispira A.St.-Hil. & Pind & 6174 \\
\hline Heliocarpus popayanensis Kunth & $\mathrm{MASu}$ & 19334 \\
\hline Hibiscus sp. * & PAçu & 37887 \\
\hline Krapovickasia sp. & Itar & 49810 \\
\hline Pachira glabra Pasq. & PAçu & 33081 \\
\hline Pavonia communis A.St.-Hil. & Jund, MASu & 37798 \\
\hline Pseudobombax grandiflorum (Cav.) A.Robyns & MASu, Ubat & 14074 \\
\hline Sida cordifolia L. * & Pind,Ubat & 5701 \\
\hline Sida linifolia Cav. * & Votu & 30559 \\
\hline Sida planicaulis Cav. * & Jund & 37733 \\
\hline Waltheria americana $\mathrm{L}$. & Pind & 4703 \\
\hline Wissadula hernandioides (L.Hér.) Garcke & RPret & 3962 \\
\hline \multicolumn{3}{|l|}{ Marantaceae } \\
\hline Ctenanthe sp. & PAçu & 29775 \\
\hline Maranta sp. * & Jund & 37745 \\
\hline Stromanthe tonckat (Aubl.) Eichler & Ubat & 4837 \\
\hline Thalia geniculata $\mathrm{L}$. & Pind & 4671 \\
\hline \multicolumn{3}{|l|}{ Marcgraviaceae } \\
\hline Marcgravia polyantha Delpino & Ubat & 5783 \\
\hline Schwartzia brasiliensis (Choisy) Bedell ex Gir.-Cañas & PAçu & 36039 \\
\hline \multicolumn{3}{|l|}{ Mayacaceae } \\
\hline Mayaca sellowiana Kunth & Itar & 49817 \\
\hline \multicolumn{3}{|l|}{ Melastomataceae } \\
\hline Clidemia hirta (L.) D.Don & Pind, Ubat & 4739 \\
\hline Clidemia urceolata DC. & Ubat & 3517 \\
\hline Leandra aurea (Cham.) Cogn. & MASu & 30067 \\
\hline Leandra australis (Cham.) Cogn. & PAçu & 29747 \\
\hline Miconia budlejoides Triana* & PAçu & 43585 \\
\hline Miconia cinnamomifolia (DC.) Naudin * & Ubat & 5332 \\
\hline Miconia cubatanensis Hoehne * & PAçu & 35424 \\
\hline Miconia discolor DC. * & Camp, MASu & 44596 \\
\hline Miconia latecrenata (DC.) Naudin * & Camp, PAçu & 32173 \\
\hline Miconia ligustroides (DC.) Naudin * & Camp & 22086 \\
\hline Miconia prasina $($ Sw.) DC. * & Ubat & 4834 \\
\hline Miconia pseudonervosa Cogn. * & Camp & 31956 \\
\hline Miconia serrulata (DC.) Naudin * & Ubat & 4838 \\
\hline Ossaea confertiflora (DC.) Triana & Camp & 32174 \\
\hline Tibouchina granulosa (Desr.) Cogn. * & MASu, PAçu & 28937 \\
\hline Tibouchina herbacea (DC.) Cogn. * & $\mathrm{MASu}$ & 30200 \\
\hline Tibouchina pulchra Cogn. * & PAçu & 29726 \\
\hline Tibouchina sebastianopolitana Cogn. * & Jund, MASu & 32977 \\
\hline Tibouchina stenocarpa (Schrank \& Mart. ex DC.) Cogn. * & Jund, MASu & 28680 \\
\hline
\end{tabular}


Tabela 2 (continuação)

Família / Espécie

Estação Experimental

Número IAC

Meliaceae

Cabralea canjerana (Vell.) Mart. *

PAçu

33036

Cedrela fissilis Vell. *

Camp, Pind

2326

Guarea guidonia (L.) Sleumer

MASu, Pind

8314

Guarea macrophylla Vahl

Trichilia catigua A.Juss. *

Camp, Ubat

32033

Trichilia clausseni C.DC. *

Pind

4751

Trichilia elegans A.Juss. *

Pind

2129

Trichilia hirta L. *, ***

Trichilia martiana C.DC. *

Camp, Jund, MASu, Votu

33006

Camp, Jaú

26932

Trichilia pallida Sw. *

Camp

32013

Trichilia silvatica C.DC. *

Camp

53550

Menispermaceae

Abuta selloana Eichler

Jund

49464

Cissampelos andromorpha DC.

RPret, Ubat

35542

Cissampelos glaberrima A.St.-Hil.

Camp, MASu

2381

Cissampelos pareira L. ***

Odontocarya acuparata Miers

Pind, Votu

7369

Jund, MASu

29552

28718

Monimiaceae

Mollinedia widgrenii A.DC.

Camp, MASu

45111

Moraceae

Brosimum gaudichaudii Trécul *

Votu

29541

Brosimum glaziovii Taub. *

Ficus hirsuta Schott*

Maclura tinctoria (L.) D.Don ex Steud.

MASu, PAçu

28998

MASu

9076

Moco

47779

Myristicaceae

Virola bicuhyba (Schott ex Spreng.) Warb. *

PAçu

49465

Myrtaceae

Blepharocalyx salicifolius (Kunth) O.Berg *

Votu

29562

Calyptranthes clusiifolia O.Berg

Calyptranthes lanceolata O.Berg

Calyptranthes lucida Mart. ex DC.

amp, MASu

41904

PAçu

33079

PAçu, Ubat

29788

Calyptranthes strigipes O.Berg

Campomanesia guaviroba (DC.) Kiaersk. *

PAçu, Ubat

29789

Adam, Ubat

47988

Campomanesia guazumifolia (Cambess.) O.Berg *

Camp, Pind

53551

Campomanesia neriiflora (O.Berg) Nied.

Campomanesia reitziana D.Legrand *

Camp, Ubat

34802

PAçu

47271

Campomanesia xanthocarpa (Mart.) O.Berg *

Camp

45112

Eugenia aurata O.Berg *

Votu

29468

Eugenia bimarginata DC. *

Itar

49833

Eugenia brevistyla D.Legrand *

Ubat

47938

Eugenia cuprea (O.Berg) Nied. *

PAçu

29776

Eugenia excelsa O.Berg*

Ubat

49553 
Tabela 2 (continuação)

\begin{tabular}{|c|c|c|}
\hline Família / Espécie & Estação Experimental & Número IAC \\
\hline Eugenia florida DC. * & Camp, Pind, Tiet & 45108 \\
\hline Eugenia francavilleana O.Berg * & Jund & 47098 \\
\hline Eugenia hiemalis Cambess. * & $\mathrm{MASu}$ & 39460 \\
\hline Eugenia leptoclada O.Berg * & Camp & 32164 \\
\hline Eugenia monosperma Vell. * & PAçu & 28901 \\
\hline Eugenia mosenii (Kausel) Sobral * & PAçu & 29779 \\
\hline Eugenia myrcianthes Nied. * & Votu & 47971 \\
\hline Eugenia neoglomerata Sobral * & PAçu & 48349 \\
\hline Eugenia neoverrucosa Sobral * & Camp & 46825 \\
\hline Eugenia paracatuana O.Berg* & Adam, Camp, Jund & 53195 \\
\hline Eugenia pisiformis Cambess. * & Ubat & 47944 \\
\hline Eugenia pluriflora DC. * & Camp, Jund & 53527 \\
\hline Eugenia prasina O.Berg * & Ubat & 47069 \\
\hline Eugenia pruinosa D.Legrand * & PAçu & 37012 \\
\hline Eugenia punicifolia (Kunth) DC. * & Itar & 49832 \\
\hline Eugenia pyriformis Cambess. * & Jund, MASu & 34660 \\
\hline Eugenia sprengelii DC. * & Jaú & 18068 \\
\hline Eugenia subavenia O.Berg* & PAçu & 36015 \\
\hline Eugenia subterminalis DC. * & PAçu & 29707 \\
\hline Eugenia sulcata Spring ex Mart. * & Jund & 38215 \\
\hline Eugenia umbellata Spreng. * & Jund & 37720 \\
\hline Eugenia uniflora L. * & MASu & 9085 \\
\hline Marlierea excoriata Mart. & Ubat & 47077 \\
\hline Marlierea tomentosa Cambess. & Ubat & 46845 \\
\hline Myrcia anacardiifolia Gardner * & PAçu & 36689 \\
\hline Myrcia bella Cambess. * & Pind & 29051 \\
\hline Myrcia egensis (O.Berg) McVaugh * & PAçu & 20714 \\
\hline Myrcia flagellaris (D.Legrand) Sobral *, **,*** & PAçu & 36684 \\
\hline Myrcia glabra (O.Berg) D.Legrand * & PAçu & 36009 \\
\hline Myrcia guianensis (Aubl.) DC. * & Camp & 29926 \\
\hline Myrcia heringii D.Legrand * & PAçu & 29778 \\
\hline Myrcia pubipetala Miq. * & PAçu & 36014 \\
\hline Myrcia spectabilis DC. * & Ubat & 47942 \\
\hline Myrcia splendens (Sw.) DC. * & Camp, Jund, PAçu, Ubat & 45105 \\
\hline Myrcia tomentosa (Aubl.) DC. * & Adam, Camp, Votu & 47974 \\
\hline Myrciaria cuspidata O.Berg * & Itar & 49830 \\
\hline Myrciaria floribunda (H.West ex Willd.) O.Berg * & Jund & 37804 \\
\hline Pimenta pseudocaryophyllus (Gomes) Landrum * & Camp, MASu & 34780 \\
\hline Plinia cauliflora (Mart.) Kausel * & Camp & 48095 \\
\hline Plinia peruviana (Poir.) Govaerts * & Ubat & 48017 \\
\hline Psidium cattleianum Sabine * & Jund, PAçu & 35288 \\
\hline Psidium guineense $\mathrm{Sw} . *$ & Jund & 30268 \\
\hline Psidium sartorianum (O.Berg) Nied. * & Camp & 46977 \\
\hline
\end{tabular}


Tabela 2 (continuação)

Família / Espécie

Estação Experimental

Número IAC

Nyctaginaceae

Guapira opposita (Vell.) Reitz *

Camp

46601

Pisonia sp.

Jund

35530

Ochnaceae

Ouratea parvifolia (A.St.-Hil.) Engl.

Jund

37743

Olacaceae

Heisteria silvianii Schwacke

Ubat

4848

Tetrastylidium grandifolium (Baill.) Sleumer

PAçu

53005

Onagraceae

Ludwigia octovalvis (Jacq.) P.H.Raven *

Ludwigia sericea (Cambess.) H.Hara *

Camp, Pinda, Pind, Ubat

36931

Camp

36925

Orchidaceae

Cleistes sp. *

PAçu

33117

Encyclia sp. *

Oeceoclades maculata (Lindl.) Lindl. *

Oncidium sp. *

PAçu

37246

Camp, RPret

4267

Pleurothallis sp. *

PAçu

33074

Sarcoglottis sp. *

PAçu

29795

Oxalidaceae

Oxalis barrelieri L. *

Jund

34655

Oxalis debilis Kunth *

Oxalis rhombeo-ovata A.St.-Hil. *

Votu

29550

PAçu

35625

Camp, Tiet

34778

Passifloraceae

Passiflora alata Curtis *

Passiflora amethystina J.C.Mikan *

Camp, Jund

37210

Passiflora capsularis L. *

Jund

31085

Passiflora cincinnata Mast. *

SRoq

7287

Passiflora edulis Sims *

Camp, Votu

33029

$\mathrm{MASu}$

32589

Passiflora foetida L.

MASu

52791

Passiflora haematostigma Mart. ex Mast. *

CBoni

31095

Passiflora morifolia Mast. *

$\mathrm{MASu}$

39538

Phyllanthaceae

Hieronyma alchorneoides Allemão *

Ubat

5324

Phytolaccaceae

Gallesia integrifolia (Spreng.) Harms

Moco

47518

Phytolacca thyrsiflora Fenzl. ex J.A.Schmidt *

Seguieria langsdorffii Moq. *

Jund, MASu, Ubat

37741

Camp, MASu

17320

Piperaceae

Peperomia corcovadensis Gardner

PAçu

35776

Peperomia glabella (Sw.) A.Dietr.

PAçu

36685

Peperomia nitida Dahlst. ***

$\mathrm{MASu}$

29017

Peperomia pseudoestrellensis C.DC.

PAçu

37234

Peperomia rotundifolia (L.) Kunth

PAçu, Ubat

36033 
Tabela 2 (continuação)

\begin{tabular}{|c|c|c|}
\hline Família / Espécie & Estação Experimental & Número IAC \\
\hline Peperomia urocarpa Fisch. \& C.A.Mey. & PAçu & 36032 \\
\hline Piper aduncum L. * & Camp, MASu, Votu & 46602 \\
\hline Piper amalago L. * & Adam, Camp, Moco & 47987 \\
\hline Piper amplum Kunth * & PAçu & 47267 \\
\hline Piper arboreum Aubl. * & $\begin{array}{c}\text { Camp, Moco, MASu, } \\
\text { PAcu, Pind, Ubat, Votu }\end{array}$ & 48217 \\
\hline Piper cernuum Vell. * & PAçu, Ubat & 47268 \\
\hline Piper claussenianum (Miq.) C.DC. * & Camp & 48097 \\
\hline Piper corcovadensis (Miq.) C.DC. * & Camp, Jund & 48216 \\
\hline Piper crassinervium Kunth * & Moco & 47513 \\
\hline Piper dilatatum Rich. * & MASu, Ubat & 47087 \\
\hline Piper gaudichaudianum Kunth * & PAçu, Ubat & 47265 \\
\hline Piper glabratum Kunth * & $\mathrm{MASu}$ & 48388 \\
\hline Piper hemmendorffii C.DC. * & Moco & 51491 \\
\hline Piper hispidinervum C.DC. * & Votu & 4857 \\
\hline Piper hispidum Sw. * & MASu, Ubat & 48391 \\
\hline Piper lepturum Kunth * & MASu & 47085 \\
\hline Piper malacophyllum (C.Presl) C.DC. * & Camp & 46798 \\
\hline Piper mollicomum Kunth * & $\begin{array}{l}\text { Camp, Jund, MASu, Pind, } \\
\text { Ubat }\end{array}$ & 53214 \\
\hline Piper permucronatum Yunck. * & Ubat & 48045 \\
\hline Piper rivinoides Kunth * & Ubat & 47078 \\
\hline Piper solmsianum C.DC. * & Ubat & 46032 \\
\hline Piper umbellatum L. * & Camp, PAçu & 46978 \\
\hline Piper xylosteoides (Kunth) Steud. *, *** & Jund, Ubat & 31152 \\
\hline \multicolumn{3}{|l|}{ Plantaginaceae } \\
\hline Achetaria ocymoides (Cham. \& Schltdl.) Wettst. & Ubat & 3513 \\
\hline Plantago sp. * & PAçu & 37232 \\
\hline Scoparia dulcis L. & Camp & 21516 \\
\hline \multicolumn{3}{|l|}{ Poaceae } \\
\hline Andropogon bicornis L. & Itar, MASu, PAçu & 49726 \\
\hline Axonopus compressus (Sw.) P.Beauv. * & Camp & 9336 \\
\hline Axonopus scoparius (Flüggé) Kuhlm. * & $\mathrm{MASu}$ & 16260 \\
\hline Bothriochloa ischaemum (L.) Keng * & $\mathrm{MASu}$ & 16263 \\
\hline Cenchrus ciliaris L. (ExIn) & Camp & 9309 \\
\hline Cenchrus clandestinus (Hochst. ex Chiov.) Morrone & Camp & 2229 \\
\hline Cenchrus echinatus L. & Camp, RPret & 11714 \\
\hline Cenchrus polystachios (L.) Morrone & Camp & 16165 \\
\hline Cenchrus purpureus (Schumach.) Morrone & Camp, MASu, RPret & 16261 \\
\hline Cenchrus setigerus Vahl & Camp & 9311 \\
\hline Chloris gayana Kunth & Camp & 16044 \\
\hline Chloris orthonoton Döll & Moco & 16248 \\
\hline Chloris pycnothrix Trin. & Camp & 152 \\
\hline Chusquea oligophylla Rupr. & PAçu & 47287 \\
\hline
\end{tabular}


Tabela 2 (continuação)

\begin{tabular}{|c|c|c|}
\hline Família / Espécie & Estação Experimental & Número IAC \\
\hline Coix lacryma-jobi L. & Camp & 5654 \\
\hline Cymbopogon densiflorus (Steud.) Stapf & Camp & 10031 \\
\hline Cynodon dactylon (L.) Pers. (ExIn) & Camp, Pind & 713 \\
\hline Eragrostis ciliaris (L.) R.Br. & Pind & 166 \\
\hline Eustachys disticophylla (Lag.) Nees & Camp & 16058 \\
\hline Hyparrhenia rufa (Nees) Stapf (ExIn) & Camp & 59 \\
\hline Ichnanthus pallens (Sw.) Munro ex Benth. & Jund, PAçu & 28678 \\
\hline Ichnanthus ruprechtii Döll & Pind & 4755 \\
\hline Lasiacis sorghoidea (Desv. ex Ham.) Hitchc. \& Chase & MASu & 24410 \\
\hline Leptochloa virgata (L.) P.Beauv. & Pind, RPret & 167 \\
\hline Merostachys skvortzovii Send. * & CBoni & 54280 \\
\hline Merostachys ternata Nees * & PAçu & 36668 \\
\hline Morronea trichidiachnis (Döll) Zuloaga \& Scataglini & $\mathrm{MASu}$ & 30138 \\
\hline Olyra glaberrima Raddi & PAçu & 35734 \\
\hline Oplismenus hirtellus (L.) P.Beauv. & Jund & 28685 \\
\hline Parodiolyra micrantha (Kunth) Davidse \& Zuloaga & Camp, MASu, PAçu & 34777 \\
\hline Paspalum acuminatum Raddi * & Camp & 22833 \\
\hline Paspalum compressifolium Swallen *, *** & MASu & 16258 \\
\hline Paspalum conjugatum P.J.Bergius * & Camp, Moco, Ubat & 16249 \\
\hline Paspalum cordatum Hack. * & Itar & 49724 \\
\hline Paspalum hyalinum Nees ex Trin. * & Itar & 49765 \\
\hline Paspalum maculosum Trin. * & Itar & 49708 \\
\hline Paspalum mandiocanum Trin. * & PAçu & 21938 \\
\hline Paspalum notatum Flüggé * & Pind & 4724 \\
\hline Paspalum oteroi Swallen *,\# & Camp, MASu & 16256 \\
\hline Paspalum paniculatum L. * & PAçu & 21939 \\
\hline Paspalum pauciciliatum (Parodi) Herter * & MASu & 16255 \\
\hline Paspalum plicatulum Michx. * & Camp & 9321 \\
\hline Paspalum polyphyllum Nees * & Itar & 49720 \\
\hline Paspalum rojasii Hack. * & Itar & 49707 \\
\hline Phyllostachys edulis (Carrière) J.Houz. & Camp & 24341 \\
\hline Pseudechinolaena polystachya (Kunth) Stapf & MASu, Ubat & 30183 \\
\hline Saccharum sp. * & Itar & 49706 \\
\hline Setaria scabrifolia (Nees) Kunth & Itar & 49712 \\
\hline Setaria sulcata Raddi & Camp, Jund & 28659 \\
\hline Stenotaphrum secundatum (Walter) Kuntze & Camp & 7774 \\
\hline \multicolumn{3}{|l|}{ Polygalaceae } \\
\hline Acanthocladus brasiliensis (Klotzsch ex A.St.-Hil. \& Moq.) Hassk. & Camp & 18902 \\
\hline Bredemeyera sp. & Jund & 38213 \\
\hline Caamembeca laureola (A.St.-Hil. \& Moq.) J.F.B.Pastore & Ubat & 4428 \\
\hline Diclidanthera sp. & Jund & 29341 \\
\hline Monnina richardiana A.St.-Hil. \& Moq. & Itar & 49841 \\
\hline Polygala lycopodioides Chodat & Itar & 49837 \\
\hline
\end{tabular}


Tabela 2 (continuação)

Família / Espécie

Polygala paniculata L.

Securidaca ovalifolia A.St.-Hil. \& Moq.

Polygonaceae

Polygonum acuminatum Kunth *

Polygonum hydropiperoides Michx. *

Polypodiaceae

Adiantum raddianum C.Presl

Adiantum tetraphyllum Humb. \& Bonpl. ex Willd.

Pontederiaceae

Eichhornia crassipes (Mart.) Solms

Portulacaceae

Portulaca oleracea L.

Primulaceae

Clavija sp.

Cybianthus cuneifolius Mart.

Geissanthus ambiguus (Mart.) G.Agostini

Lysimachia arvensis (L.) U.Manns \& Anderb.

Myrsine balansae (Mez) Arechav. *

Myrsine coriacea (Sw.) R.Br. ex Roem. \& Schult. *

Myrsine gardneriana A.DC. *

Myrsine hermogenesii (Jung-Mend. \& Bernacci) M.F.

Freitas \& Kin.-Gouv. *

Myrsine leuconeura Mart. *

Myrsine umbellata Mart. *

Stylogyne laevigata (Miq.) Mez

Stylogyne lhotzkyana (A.DC.) Mez

Stylogyne warmingii Mez

Proteaceae

Roupala montana Aubl. var. brasiliensis (Klotzsch) K.S.Edwards

Ranunculaceae

Clematis dioica L. *

Rhamnaceae

Colubrina glandulosa Perkins *

Gouania ulmifolia Hook. \& Arn. ***

Rhamnidium elaeocarpum Reissek

Rhamnus sphaerosperma Sw.

Rosaceae

Prunus myrtifolia (L.) Urb. *

Rubus urticifolius Poir. *

Rubiaceae

Alibertia concolor (Cham.) K.Schum. *

Alseis floribunda Schott

Amaioua sp.

\author{
Estação Experimental \\ PAçu, Ubat \\ Votu \\ MASu \\ Pinda \\ $\mathrm{MASu}$ \\ RPret \\ Jund, MASu \\ Camp \\ Adam \\ Jund \\ Camp, MASu, PAçu, Pind \\ Itar, MASu \\ $\mathrm{MASu}$ \\ Jund, MASu, PAçu, SRoq, \\ Ubat \\ Camp \\ Ubat \\ Votu \\ Jund, MASu \\ Moco, PAçu \\ Moco, PAçu \\ Camp,Jund
}

Número IAC

47279

30575

7351

9350

7358

2291

30210

4422

30855

35283

40533

45533

39466

36329

31974

44035

48051

9068

49654

49654

47507

Camp, MASu, Pind

19949

Jund, Ubat

28671

Camp, MASu

29216

MASu

42752

Votu

29453

Itar

50217

PAçu

36671

SRoq, Ubat

28788

Jund

33720

Ubat

22439

PAçu 
Tabela 2 (continuação)

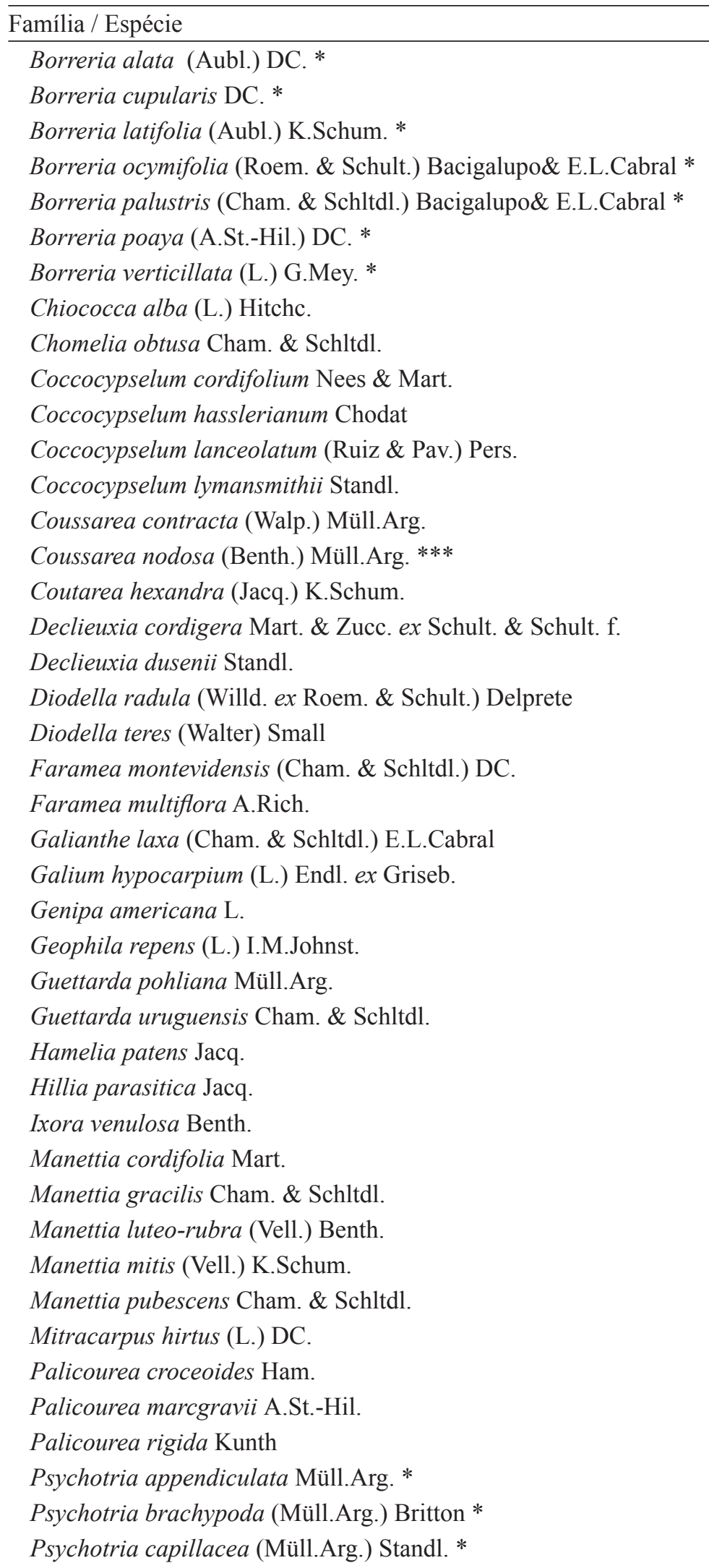

\begin{tabular}{|c|c|}
\hline Estação Experimental & Número IAC \\
\hline Ubat & 5407 \\
\hline Jund & 32990 \\
\hline PAçu & 29722 \\
\hline PAçu, Ubat & 35748 \\
\hline PAçu & 35997 \\
\hline Itar & 50497 \\
\hline Jund, MASu & 35297 \\
\hline Jund, MASu, Votu & 28740 \\
\hline Camp, Votu & 32018 \\
\hline PAçu, Ubat & 36021 \\
\hline Jund, PAçu, Ubat & 34116 \\
\hline Camp, Itar, Jund, Ubat & 31966 \\
\hline Itar & 50495 \\
\hline Pind & 28231 \\
\hline Ubat & 28230 \\
\hline MASu, Tiet & 30185 \\
\hline Itar & 50499 \\
\hline Itar & 32297 \\
\hline Ubat & 28551 \\
\hline Pind & 372 \\
\hline PAçu & 48364 \\
\hline PAçu & 32264 \\
\hline Jund & 30243 \\
\hline MASu, SRoq, Votu & 39457 \\
\hline Camp, PAçu & 7933 \\
\hline PAçu, Pind & 33101 \\
\hline Camp & 53525 \\
\hline Camp & 53526 \\
\hline MASu & 17780 \\
\hline Ubat & 48487 \\
\hline Camp, Jund, MASu, Tiet & 34808 \\
\hline Camp, Tiet, Votu & 44589 \\
\hline Camp, Jund, MASu & 33026 \\
\hline Jund, MASu, SRoq & 28732 \\
\hline Ubat & 3528 \\
\hline $\mathrm{MASu}$ & 7363 \\
\hline Camp, Jund & 19805 \\
\hline PAçu & 29750 \\
\hline Jund & 37359 \\
\hline Pind & 48103 \\
\hline Jund & 33724 \\
\hline PAçu & 32263 \\
\hline Adam & 30875 \\
\hline
\end{tabular}


Tabela 2 (continuação)

\begin{tabular}{|c|c|c|}
\hline Família / Espécie & Estação Experimental & Número IAC \\
\hline Psychotria capitata Ruiz \& Pav. *, *** & Pind & 6289 \\
\hline Psychotria carthagenensis Jacq. * & $\begin{array}{l}\text { Adam, Camp, Jund, } \\
\text { MASu, Pind, RPret, Votu }\end{array}$ & 30889 \\
\hline Psychotria deflexa DC. * & Jund, PAçu, Pind, Ubat & 36343 \\
\hline Psychotria hastisepala Müll.Arg. * & Jund, MASu & 37729 \\
\hline Psychotria hoffmannseggiana (Willd. ex Schult.) Müll.Arg. * & Pind, Ass & 53563 \\
\hline Psychotria leiocarpa Cham. \& Schltdl. * & Jund, MASu, PAçu & 38214 \\
\hline Psychotria longipes Müll.Arg. * & Ubat & 44033 \\
\hline Psychotria lupulina Benth. * & Jund, MASu & 37375 \\
\hline Psychotria mapourioides DC. * & Jund,PAçu & 33719 \\
\hline Psychotria nemorosa Gardner * & PAçu & 40637 \\
\hline Psychotria nuda (Cham. \& Schltdl.) Wawra * & Ubat & 30773 \\
\hline Psychotria patentinervia Müll.Arg. * & Ubat & 28294 \\
\hline Psychotria stachyoides Benth. * & Jund & 37363 \\
\hline Psychotria suterella Müll.Arg. * & PAçu & 32261 \\
\hline Psychotria trichophora Müll.Arg. * & PAçu & 48348 \\
\hline Psychotria vellosiana Benth. * & Jund, MASu, Ubat & 37812 \\
\hline Randia armata (Sw.) DC. & Tiet, Votu & 29129 \\
\hline Randia calycina Cham. & Votu & 29474 \\
\hline Richardia brasiliensis Gomes & Pind, RPret & 3413 \\
\hline Rudgea coriacea (Spreng.) K.Schum. & PAçu & 32266 \\
\hline Rudgea jasminoides (Cham.) Müll.Arg. & PAçu & 35638 \\
\hline Rudgea nobilis Müll.Arg. **, *** & Ubat & 5323 \\
\hline Rudgea recurva Müll.Arg. & PAçu & 48345 \\
\hline Rudgea viburnoides (Cham.) Benth. & Votu & 30555 \\
\hline Sabicea villosa Willd. ex Schult. \# & PAçu & 37914 \\
\hline Simira viridiflora (Allemão \& Saldanha) Steyerm. *** & Ubat & 19557 \\
\hline Tocoyena sp. & Votu & 30560 \\
\hline \multicolumn{3}{|l|}{ Rutaceae } \\
\hline Angostura sp. & Tiet & 29167 \\
\hline Balfourodendron riedelianum (Engl.) Engl. * & Pind & 2128 \\
\hline Esenbeckia febrifuga (A.St.-Hil.) A.Juss. ex Mart. * & Camp & 32019 \\
\hline Esenbeckia grandiflora Mart. * & MASu, PAçu & 39854 \\
\hline Esenbeckia leiocarpa Engl. * & Tiet & 29125 \\
\hline Galipea jasminiflora (A.St.-Hil.) Engl. & Camp, Jund & 47508 \\
\hline Helietta apiculata Benth. * & Adam, Pind & 47989 \\
\hline Metrodorea nigra A.St.-Hil. & Camp, MASu & 46826 \\
\hline Metrodorea stipularis Mart. & Camp & 44005 \\
\hline Pilocarpus sp. ${ }^{*}$ & Ubat & 30772 \\
\hline Zanthoxylum caribaeum Lam. * & Camp, MASu & 46827 \\
\hline Zanthoxylum fagara (L.) Sarg. * & MASu, Pind & 9081 \\
\hline Zanthoxylum monogynum A.St.-Hil. * & Pind & 2125 \\
\hline Zanthoxylum petiolare A.St.-Hil. \& Tul. *, **, *** & Votu & 47962 \\
\hline
\end{tabular}


Tabela 2 (continuação)

\begin{tabular}{l}
\hline Família / Espécie \\
\hline Zanthoxylum rhoifolium Lam. * \\
Zanthoxylum riedelianum Engl. * \\
Sabiaceae \\
Meliosma sellowii Urb. \\
Salicaceae \\
Abatia americana (Gardner) Eichler \\
Casearia decandra Jacq. * \\
Casearia mariquitensis Kunth * \\
Casearia obliqua Spreng. * \\
Casearia rupestris Eichler * \\
Casearia sylvestris Sw. * \\
Prockia crucis P.Browne ex L. \\
Xylosma venosa N.E.Br.
\end{tabular}

Estação Experimental
Jund, PAçu, Votu
Camp, Moco, Votu
PAçu

Número IAC

28672

31951

Santalaceae

Phoradendron affine (Pohl ex DC.) Engl. \& Krause

Jund

35671

Phoradendron berteroanum (DC.) Griseb.

Phoradendron chrysocladon A.Gray

$$
\text { PAçu }
$$

21409

Votu

35727

MASu, PAçu

29559

Votu

9087

29452

Camp, Jund, PAçu, Tiet,

Ubat, Votu

53535

Pind, Votu

4639

Votu

29489

Camp

33013

Camp

5279

PAçu

53022

Sapindaceae

Allophylus edulis (A.St.-Hil. et al.) Hieron. ex Niederl. *

Camp, PAçu

32020

Allophylus petiolulatus Radlk. *

PAçu

35670

Cardiospermum sp.

Camp, MASu

28751

Cupania oblongifolia Mart. *

PAçu

35667

Cupania racemosa (Vell.) Radlk. *

MASu

14070

Cupania vernalis Cambess. *

Dodonaea sp. *

Camp, Jund, MASu, Tiet

46969

Matayba guianensis Aubl. *

SRoq

28794

Paullinia carpopoda Cambess. *

Votu

29455

Paullinia meliifolia Juss. *

Ubat

5641

Paullinia trigonia Vell. *

PAçu

35680

Serjania communis Cambess. *

Camp

44570

Serjania fuscifolia Radlk. *

Serjania meridionalis Cambess. *

PAçu

35459

Camp, PAçu

49105

Camp

49107

Serjania multiflora Cambess. *

Camp

44593

Serjania ovalifolia Radlk. *

Pind

4685

Urvillea ulmacea Kunth

Camp

44595

Sapotaceae

Chrysophyllum flexuosum Mart.

PAçu

35683

Chrysophyllum marginatum (Hook. \& Arn.) Radlk.

Votu

30549

Pouteria caimito (Ruiz \& Pav.) Radlk. *

PAçu, Ubat

48311

Pouteria venosa (Mart.) Baehni *

PAçu

22153

Pradosia lactescens (Vell.) Radlk.

PAçu

35504 
Tabela 2 (continuação)

Família / Espécie

Estação Experimental

Número IAC

Schizaeaceae

Lygodium volubile $\mathrm{Sw}$.

Ubat

Scrophulariaceae

Buddleja stachyoides Cham. \& Schltdl. *

Itar

50219

Selaginellaceae

Selaginella sulcata (Desv. ex Poir.) Spring

Ubat

5782

Siparunaceae

Siparuna brasiliensis (Spreng.) A.DC. *

Ubat

46834

Siparuna guianensis Aubl. *

Camp, Moco, MASu, Ubat

53538

Smilacaceae

Smilax elastica Griseb. *

PAçu

35628

Smilax fluminensis Steud. *

RPret

49334

Solanaceae

Acnistus arborescens (L.) Schltdl. *

Ubat

30791

Athenaea picta (Mart.) Sendtn.

Ubat

3490

Aureliana fasciculata (Vell.) Sendtn.

PAçu

35495

Brunfelsia uniflora (Pohl) D.Don

PAçu

35496

Calibrachoa micrantha (R.E.Fr.) Stehmann \& Semir

Itar

22773

Capsicum baccatum L. var. praetermissum (Heiser \&

P.G.Smith) Hunz. *

Capsicum flexuosum Sendtn. *

$\mathrm{MASu}$

38913

Capsicum frutescens L. *

Ubat

4390

Capsicum mirabile Mart. *

Pind

24638

Cestrum intermedium Sendtn.

PAçu

35784

Cestrum mariquitense Kunth

MASu, SRoq

29027

Cestrum schlechtendalii G.Don

Camp, MASu, Pind

28748

Physalis sp. *

$\mathrm{MASu}$

28964

Pind

4712

Sessea sp.

Jund, Ubat

28668

Solanum aculeatissimum Jacq. *

Solanum americanum Mill. *

Solanum atropurpureum Schrank *

Camp, Pind, Pinda

19154

Camp, Jund

44576

Solanum cinnamomeum Sendtn. *

Pind

4691

Solanum didymum Dunal *

PAçu

35647

Solanum diploconos (Mart.) Bohs *

Camp

36435

Solanum hexandrum Vell. *

PAçu

47277

Solanum mammosum L. *

Ubat

48023

Solanum mauritianum Scop. *

Jund

40118

Solanum oocarpum Sendtn. *

PAçu, SRoq

35454

Solanum paniculatum L. *

Solanum pseudoquina A.St.-Hil. *

Camp, Pind

32165

Camp, Ubat

32168

Solanum pycnanthemum Mart. *

Itar

50220

Solanum scuticum M.Nee*

Pind

24932

Solanum swartzianum Roem. \& Schult. *

Pind

52845

PAçu

35494 
Tabela 2 (continuação)

Família / Espécie
Solanum vaillantii Dunal *

Solanum viarum Dunal *

$\begin{array}{cc}\text { Estação Experimental } & \text { Número IAC } \\ \text { Ubat } & 23152 \\ \text { Pind } & 4668\end{array}$

Styracaceae

Styrax acuminatus Pohl

PAçu

35657

Styrax camporum Pohl

Styrax ferrugineus Nees \& Mart.

Camp, Jund, MASu, Votu

53544

Styrax latifolius Pohl

Camp

21831

MASu

8302

Styrax pohlii A.DC.

Camp

21505

Symplocaceae

Symplocos estrellensis Casar.

PAçu

35654

Symplocos laxiflora Benth.

PAçu

36046

Symplocos trachycarpos Brand

PAçu

35464

Trigoniaceae

Trigonia eriosperma (Lam.) Fromm \& Santos \#

Jund

28704

Trigonia nivea Cambess.

Camp, MASu, PAçu

30169

Urticaceae

Boehmeria cylindrica (L.) Sw. *

Pind

4670

Cecropia pachystachya Trécul *

Camp

53539

Coussapoa microcarpa (Schott) Rizzini

PAçu

29096

Pilea pubescens Liebm.

SRoq

28787

Pourouma sp. *

PAçu

37905

Urera baccifera (L.) Gaudich. ex Wedd.

Camp, PAçu, Pind

36676

Verbenaceae

Aloysia virgata (Ruiz \& Pav.) Juss.

Camp

46966

Citharexylum myrianthum Cham.

Lantana camara L. *

Lantana hypoleuca Briq. *

Lantana trifolia L. *

Lantana undulata Schrank*

Lippia brasiliensis (Link) T.R.S.Silva *

Lippia lippioides (Cham.) Rusby *

Moco, PAçu

50815

Camp, MASu, PAçu,

RPret

44581

Pind

4666

MASu, SRoq

30088

Ubat

5632

$\mathrm{MASu}$

30112

Camp

46830

Lippia lupulina Cham. *

Itar

49756

Lippia origanoides Kunth *

Stachytarpheta cayennensis (Rich.) Vahl

Pinda

48396

Stachytarpheta polyura Schauer \#

Pind

3421

Ubat

4826

Violaceae

Anchietea pyrifolia (Mart.) G.Don

MASu, Ubat

42480

Hybanthus atropurpureus (A.St.-Hil.) Taub.

Hybanthus bigibbosus (A.St.-Hil.) Hassl.

Hybanthus communis (A.St.-Hil.) Taub.

Camp, Jund, MASu

33001

Camp, Tiet

44571

Pind

24639

Vitaceae

Cissus erosa Rich.

SRoq

7286

Cissus verticillata (L.) Nicolson \& C.E.Jarvis

PAçu, Sroq

35460 
Tabela 2 (continuação)

\begin{tabular}{lcc}
\hline Família / Espécie & Estação Experimental & Número IAC \\
\hline Vochysiaceae & & \\
Callisthene castellanosii H.F.Martins & MASu & 14076 \\
Callisthene minor Mart. & Jund & 21355 \\
Qualea grandiflora Mart. & Votu & 29461 \\
Qualea multiflora Mart. & Votu & 29497 \\
Qualea multiflora subsp. pubescens (Mart.) Stafleu & Pind & 4695 \\
Vochysia bifalcata Warm. * & PAçu & 35631 \\
Vochysia laurifolia Warm. * & Ubat & 4827 \\
Vochysia magnifica Warm. * & MASu \\
Winteraceae & & 8304 \\
Drimys brasiliensis Miers * & Itar & \\
Xyridaceae & & 49809 \\
Xyris sp. & Itar & 49819 \\
Zingiberaceae & & \\
Hedychium coronarium J.Koenig & MASu, Ubat & 30204 \\
\hline
\end{tabular}

geram dúvida sobre a adequação de estarem na lista de espécies ameaçadas.

Walter et al. (2005a) relataram mais de 60 famílias que apresentam espécies cultivadas (domesticadas, semi-domesticadas e não domesticadas) com ocorrência nativa, subespontânea ou com raças locais no Brasil, o que evidencia o potencial brasileiro nestas áreas para a exploração e o melhoramento de novos produtos para a alimentação, medicina, floricultura, cosméticos, fibras, produção de energia, dentre outros.

O Brasil tem muitas espécies frutíferas nativas que possuem potencial para serem transformadas em fruteiras de expressão econômica (Clement 2001), com destaque, em nível mundial, para o abacaxi (Ananas) e plantas de outros gêneros, tais como Annona (Carvalho et al. 2000), Passiflora (Faleiro et al. 2005), Psidium (Santos et al. 2008) e Rubus. Já a floricultura no Brasil ainda é pouco explorada, porém o país tem plantas nativas atrativas como bromélias, antúrios, orquídeas e helicônias, que são menos empregadas no setor ornamental por diversos fatores como desinformação e falta de pesquisa e/ou divulgação (Lorenzi \& Souza 2008). Outra utilização das espécies nativas com potencial ornamental é para arborização urbana. Atualmente as espécies arbustivas/árboreas utilizadas na maioria das cidades brasileiras são pouco diversificadas e ocorre uma grande utilização de espécies exóticas (Kinoshita et al. 2006). O uso de espécies nativas deveria ser estimulado, pois permite que moradores da região conheçam e valorizem a flora local, além de proporcionar benefícios para a fauna nativa.

A identificação de espécies nativas no Estado de São Paulo que não estão assim mencionadas na Lista de Espécies da Flora do Brasil reflete a necessidade constante de troca de informações entre os botânicos brasileiros e a relevância de consultas frequentes às coleções disponíveis nos herbários.

As espécies exóticas invasoras encontradas nos fragmentos precisam ser monitoradas e controladas, tendo em vista que promovem modificações nos ambientes naturais: ciclagem de nutrientes e produtividade vegetal, facilitação de propagação de focos de incêndios devido ao acúmulo de biomassa, alteração nas relações entre espécies nativas e seus polinizadores, representando assim, um grave problema para o funcionamento dos ecossistemas (GISP 2005, Zenni \& Ziller 2011). O potencial de modificação dos ambientes naturais pelas espécies exóticas é tão grande que as plantas invasoras atualmente são consideradas a segunda maior ameaça à biodiversidade (Ziller 2001). Em nosso país, existem poucos estudos sobre invasão biológica em áreas protegidas, provavelmente porque os impactos causados são relativamente lentos (Freitas 1999, Martins et al. 2001). Embora ocorram espécies exóticas prejudiciais existem aquelas com reduzido potencial de invasão e que podem atingir uma condição de naturalização, reproduzindo-se e mantendo-se no ambiente; mas, devido a limitações na dispersão ou na competência ecológica, essas espécies 
ficam restritas a áreas próximas do local de introdução, sem ocupar novas áreas naturais (Moro et al. 2012). Algumas dessas espécies exóticas naturalizadas podem ser úteis, tais como a Momordica charantia, indicada como medicinal na Relação Nacional de Plantas Medicinais de Interesse ao Sistema Único de Saúde.

Primack \& Rodrigues (2006) propuseram o uso de três critérios para estabelecer prioridades de conservação das espécies e comunidades: 1) critério de diferenciação, baseado em ocorrência de espécies endêmicas raras; 2) perigo, baseado na ocorrência de espécies ameaçadas de extinção; e 3) utilidade, baseado na prioridade de conservação de espécies com valor atual ou potencial. Na maioria dos remanescentes das EEx, pode-se aplicar ao menos um desses critérios. Dessa forma recomenda-se a preservação das áreas remanescentes aliada à adequação à legislação vigente relativa às áreas de preservação permanente e de reserva legal. Recomenda-se, fortemente, a ampliação dos mecanismos de proteção de todas as áreas como, por exemplo, o seu tombamento pelo CONDEPHAAT (Conselho Estadual de Defesa do Patrimônio Histórico, Arqueológico, Artístico e Turístico do Estado de São Paulo) e pelos municípios.

\section{Agradecimentos}

A todos os funcionários do Instituto Agronômico de Campinas, em especial àqueles do Herbário IAC, que direta ou indiretamente contribuíram para a realização deste trabalho; aos colegas das Estações Experimentais, pelas informações sobre as áreas nativas.

\section{Literatura citada}

Aguiar, A.T.E., Gonçalves, C., Paterniani, M.E.A.G.Z., Tucci, M.L.S.A. \& Castro, C.E.F. (eds.). 2014. Instruções agrícolas para as principais culturas econômicas. Instituto Agronômico, Campinas. 7 ed.

Almeida, D.S. 1998. Recuperação ecológica de paisagens fragmentadas. Série Técnica IPEF 12: 99-104.

Benzing, D.H. 1980. The biology of the Bromeliads. Mad River Press, Eureka, California.

Bernacci, L.C., Franco, G.A.D.C., Árbocz, G.F., Catharino, E.L.M., Durigan, G. \& Metzger, J.P. 2006. O efeito da fragmentação florestal na composição e riqueza de árvores na região da reserva Morro Grande (Planalto Ibiúna, SP). Revista Instituto Florestal 18: 121-166.
Capozzoli, U. (ed.). 2009. História da ciência no Brasil 2: o envolvimento com o átomo: de 1921 a 1969: da visita de Einstein à investigação espacial. Scientific American Brasil. Duetto, São Paulo, pp. 13-15.

Carvalho, J.E.U., Ribeiro, M.A.C., Nascimento, W.M.O. \& Muller, C.N. 2000. Enxertia de gravioleira (Annona muricata L.) em porta-enxertos dos gêneros Annona e Rollinia. Embrapa - Amazônia Oriental: Comunidado Técnico ${ }^{\circ} 27$, pp. 1-4.

Carvalho, M.B., Bernacci, L.C. \& Coelho, R.M. 2013. Floristic and phytosociology in a physiognomic gradient of riverine forest in Cerrado, Campinas, SP. Biota Neotropica 13: 110-120.

Cerqueira, R., Brant, A., Nascimento, M.T. \& Pardini, R. 2003. Fragmentação: alguns conceitos. In: D.M. Rambaldi \& D.A.S. Oliveira (orgs.). Fragmentação de ecossistemas: causas, efeitos sobre a biodiversidade e recomendações de políticas públicas. Ministério do Meio Ambiente, Secretaria de Biodiversidade e Florestas, Brasília, pp. 13-14.

Chautems, A.; Araújo, A.O.; Sfair, J.C.; Barros, F.S.M., Fernandez, E.P., Moraes, M.M.V., Pessoa, S.V.A., Kutschenko, D.C.\& Messina, T. 2013. Gesneriaceae. In: G. Martinelli \& M.A. Moraes (orgs.). Livro vermelho da flora do Brasil. Instituto de Pesquisas Jardim Botânico do Rio de Janeiro, Rio de Janeiro. pp. 553-564.

Clement, C.R. 2001. Melhoramento de espécies nativas. In: L.L Nass, A.C.C. Valois, I.S. Melo \& M.C. ValadaresInglis (eds.). Recursos genéticos \& melhoramento: plantas. Fundação de Apoio à Pesquisa Agropecuária de Mato Grosso, Rondonópolis, pp. 423-441.

Código Florestal Brasileiro. 2012. Lei $\mathrm{n}^{\circ} 12.651$, de 25 de maio de 2012. Disponível em http://www2.camara. leg.br/legin/fed/lei/2012/lei-12651-25-maio-2012613076-publicacaooriginal-136199-pl.html (acesso em III-2014).

Dean, W. 1996. A ferro e fogo - a história e a devastação da Mata Atlântica brasileira. Companhia das Letras, São Paulo.

Durigan, G., Franco, G.A.D.C. \& Siqueira, M.F. 2004. A vegetação dos remanescentes de cerrado no Estado de São Paulo. In: M.D. Bitencourt \& R.R. Mendonça (orgs.).Viabilidade de conservação dos remanescentes de cerrado no Estado de São Paulo. São Paulo: Annablume, FAPESP, São Paulo.

Faleiro, F.G., Junqueira, N.T.V. \& Braga, M.F. (eds.). 2005. Maracujá: germoplasma e melhoramento genético. 1 ed. Embrapa Cerrados, Planaltina.

FAPESP. 2006. Olhar amplo sobre a biodiversidade. FAPESP, São Paulo. Disponível em http://www.fapesp. br/publicacoes/biodiversidade_port.pdf (acesso em 20-I-2014).

Ferreira, I.C.M., Coelho, R.M., Torres, R.B. \& Bernacci. L.C. 2007. Solos e vegetação nativa remanescente no município de Campinas. Pesquisa Agropecuária Brasileira 42: 1319-1327. 
Flint-Garcia, S.A. 2013. Genetics and consequences of crop domestication. Journal of Agricultural and Food Chemistry 61: 8267-8276.

Forzza, R.C., Baumgratz, J.F.A., Bicudo, C.E.M., Canhos, D.A.L., Carvalho JR. A.A., Coelho, M.A.N., Costa, A.F., Costa, D.P., Hopkins, M.G., Leitman, P.M., Lohmann, L.G., Lughadha, E.N., Maia, L.C., Martinelli, G., Menezes, M., Morim, M.P., Peixoto, A.L., Pirani, J.R., Prado, J., Queiroz, L.P., Souza, S., Souza, V.C., Stehmann, J.R., Sylvestre, L.S., Walter, B.M.T. \& Zappi, D.C. 2012. New Brazilian floristic list highlights conservation challenges. BioScience 62: $39-45$.

Freitas, G.K. 1999. Invasão biológica pelo capim-gordura (Melinis minutiflora Beauv.) em um fragmento de cerrado (A.R.I.E. Cerrado Pé-de-Gigante, Santa Rita do Passa Quatro, SP). Dissertação de Mestrado. Universidade de São Paulo, São Paulo.

GISP - Programa Global de Espécies Invasoras. 2005. América do Sul invadida: a crescente ameaça das espécies exóticas invasoras. Programa Global de Espécies Invasoras, Curitiba.

Ivanauskas, N.M., Monteiro, R. \& Rodrigues, R.R. 2001. Levantamento florístico de trecho de Floresta Atlântica em Pariquera-Açu, São Paulo, Brasil. Naturalia 26: 97-129.

Judd, W.S., Campbell, C.S., Kellogg, E.A., Stevens, P.F. \& Donoghue, M.J. 2009. Sistemática vegetal: um enfoque filogenético. Artmed, Porto Alegre.

Kinoshita, L.S., Torres, R.B., Tamashiro, J.Y., ForniMartins, E. R., Ahn, Y.J., Constâncio, S.S. \& Spinelli, T. 2006. Caminhando pelo entorno: conhecendo as plantas. In: L. S. Kinoshita, R.B. Torres, J.Y. Tamashiro \& E.R. Forni-Martins (orgs.). A Botânica no ensino básico: relatos de uma experiência transformadora. RiMa, São Carlos. pp. 51-60.

Leite, L.L. \& Coradin, L. 2011. Introdução. In: L. Coradin, A. Siminski \& A. Reis (eds.). Espécies nativas da flora brasileira de valor econômico atual ou potencial Plantas para o futuro - Região Sul. Ministério do Meio Ambiente, Brasília. pp. 17-24.

Lima, H.C., Souza, E.R., Tozzi, A.M.G.A., Perez, A.P.F., Flores, A.S., Sartori, A.L.B., Vaz, A.M.S.F., Filardi, F., Fernandes, F.M., Garcia, F.C.P., Iganci, J.R.V., Fernandes, J.M., Valls, J.F.M., Lima, L.C.P., Costa, L.C., Queiroz, L.P., Morim, M.P., Nunes, N.L.A., Queiroz, R.T., Miotto, S.T.S., Dutra, V.F., Mansano, V.F., Souza, V.C., Messina, T., Kutschenko, D.C., Penedo, T.S.A. \& Valente, A.M. 2013. Fabaceae/ Leguminosae. In: G. Martinelli \& M.A. Moraes (orgs.). Livro vermelho da flora do Brasil. Instituto de Pesquisas Jardim Botânico do Rio de Janeiro, Rio de Janeiro. 1 ed., pp. 516-548.
Lorenzi, H. \& Souza, H.M. 2008. Plantas ornamentais no Brasil. 4 ed. Instituto Plantarum de Estudos da Flora Ltda., Nova Odessa.

Martinelli, G. \& Moraes, M.A. (orgs.). 2013. Livro vermelho da flora do Brasil. Instituto de Pesquisas Jardim Botânico do Rio de Janeiro, Rio de Janeiro. 1 ed.

Martinelli, G., Valente,A.S.M., Maurenza, D., Kutschenko, D.C., Judice, D.M., Silva, D.S., Fernandez, E.P., Martins, E.M., Barros, F.S.M., Sfair, J.C.,Santos Filho, L.A.F., Abreu, M.B., Moraes, M.A., Monteiro, N.P., Pietro, P.V., Fernandes, R.A., Hering, R.L.O., Messina, T. \& Penedo, T.S.A. 2013. Avaliações de risco de extinção de espécies da flora brasileira. In: G. Martinelli \& M.A. Moraes (orgs.). Livro vermelho da flora do Brasil. Instituto de Pesquisas Jardim Botânico do Rio de Janeiro, Rio de Janeiro. pp. 60-103.

Martins, C.R., Leite, L.L. \& Haridasan, M. 2001. Recuperação de uma área degradada pela mineração de cascalho com uso de gramíneas nativas. Revista Árvore 25: 157-166.

Moro, M.F., Souza, V.C., Oliveira-Filho, A.T., Queiroz, L.P., Fraga, C.N., Rodal, M.J.N., Araújo, F.S. \& Martins, F.R. 2012. Alienígenas na sala: o que fazer com espécies exóticas em trabalhos de taxonomia, florística e fitossociologia? Acta Botanica Brasilica 26: 991-999.

Nass, L.L. \& Sigrist, M.S. 2009. Espécies silvestres: potencial de exploração via pré-melhoramento. In: A. Borém, M.T.G. Lopes \& C.R. Clement (eds.). Domesticação e melhoramento: espécies amazônicas. Universidade Federal de Viçosa, Viçosa. pp. 101-116.

Olifiers, N. \& Cerqueira, R. 2006. Fragmentação de habitat: efeitos históricos e ecológicos. In: C.F.D. Rocha, H.G. Bergallo, M.V. Sluys \& M.A.S. Alves. (eds.). Biologia da Conservação: essências. RiMa, São Carlos. pp. 261-280.

Primack, R.B. \& Rodrigues, E. 2006. Biologia da conservação. 1 ed. Planta, Londrina.

Rodrigues, R.R., Torres, R.B., Mattthes, L.A.F. \& Penha, A.S. 2004. Tree species sprouting from root buds in a semideciduous forest affected by fires. Arquivos de Biologia e Tecnologia 47: 127-133.

Sakai, E. \& Lepsch, I.F. 1984. Levantamento pedológico detalhado da Estação Experimental de Pariquera-Açu. Boletim Técnico do Instituto Agronômico 83: 1-56.

Sampaio, M.T.F, Polo, M. \& Barbosa, W. 2012. Estudo do crescimento de espécies de árvores semidecíduas em uma área ciliar revegetada. Revista Árvore 36: 879-885.

Santos, C.A.F., Castro, J.M.C., Souza, F.F., Vilarinho, A.A., Ferreira, F.R., Pádua, J.G., Borges, R.M.E., Barbieri, R.L., Souza, A.G.C. \& Rodrigues, M.A. 2008. Preliminary characterization of Psidium germplasm in different Brazilian ecogeographic regions. Pesquisa Agropecuaria do Brasil 43: 437-440.

Santos, G.M. 2005. A pesquisa científica paulista em biociências: à margem das instituições. História, Ciências, Saúde - Manguinhos 12: 51-67. 
Scaramuzza, C.A.M. 2006. Flora e ecologia dos campos de Itararé, São Paulo, Brasil. Tese de Doutorado. Universidade de São Paulo, São Paulo.

Secretaria do Meio Ambiente do Estado de São Paulo. 2004 Resolução SMA-48, de 21-IX-2004. Lista de espécies da flora ameaçadas de extinção no estado de São Paulo. Diário Oficial do Estado de São Paulo, Poder Executivo, São Paulo, 22-IX-2004. Seção I, v.114, n. 179, pp. 26-29.

Silva, A.F. \& Leitão Filho, H.F. 1982. Composição florística e estrutura de um trecho da mata atlântica de encosta no município de Ubatuba (São Paulo, Brasil). Revista Brasileira de Botânica 5: 43-52

Silva, L.A. \& Soares, J.J. 2003. Composição florística de um fragmento de floresta estacional semidecídua no município de São Carlos-SP. Revista Árvore 17: 647-656.

Souza, V.C. \& Lorenzi, H. 2008. Botânica sistemática: guia ilustrado para identificação das famílias de fanerógamas nativas e exóticas no Brasil, baseado em APG II. 3 ed. Instituto Plantarum de Estudos da Flora Ltda., Nova Odessa.

Stehmann, J.R., Forzza, R.C., Salino, A., Sobral, M., Costa, D.P. \& Kamino, L.H.Y. 2009. Plantas da Floresta Atlântica. Instituto de Pesquisas Jardim Botânico do Rio de Janeiro, Rio de Janeiro.

Tannus, J.L.S. 2007. Estudo da vegetação dos campos úmidos de Cerrado: Aspectos florísticos e ecológicos. Tese de Doutorado, Universidade Estadual Paulista, Rio Claro.

Teixeira Neto, E.A. 2009. Análise das alterações temporais dos fragmentos de vegetação no Centro Experimental do Instituto Agronômico, Campinas, utilizando técnicas de sensoriamento remoto e geoprocessamento. Trabalho de Conclusão de Curso, Universidade Estadual Paulista, Rio Claro.

Torres, R.B., Matthes, L.A.F. \& Rodrigues, R.R. 1992. Espécies florestais nativas para plantio em áreas de brejo. O Agronômico 44: 13-16.

Torres, R.B., Matthes, L.A.F. \& Rodrigues, R.R. 1994. Florística e estrutura do componente arbóreo de mata de brejo, Campinas, SP. Revista Brasileira de Botânica 17: 189-194.

Viana, V.M. 1995. Conservação da biodiversidade de fragmentos de florestas tropicais em paisagens intensivamente cultivadas. In: Conservation International do Brasil, Universidade Federal de Minas Gerais, University of Florida. Abordagens interdisciplinares para a conservação da biodiversidade e dinâmica do uso da terra no novo mundo. CI Brasil, UFMG, UFL, Belo Horizonte, Gainesville. pp. 135-154.

Victor, M.A.M., Cavalli, A.C., Guillaumon, J.R. \& Serra Filho R. 2005. Cem anos de devastação: revisitada 30 anos depois. Ministério do Meio Ambiente, Secretaria de Biodiversidade e Florestas, Brasília.

Walter, B.M.T., Cavalcanti, T.B., Bianchetti, L.B. \& Valls, J.F.M. 2005a. Origens da agricultura, centros de origem e diversificação das plantas cultivadas. In: B.M.T. Walter \& T.B. Cavalcanti (eds.). Fundamentos para a coleta de germoplasma vegetal. EMBRAPA Recursos Genéticos e Biotecnologia, Brasília.
Walter, B.M.T., Cavalcanti, T.B., Bianchetti, L.B. \& Valls, J.F.M. 2005b. Coleta de germoplasma vegetal: relevância e conceitos básicos. In: B.M.T. Walter \& T.B. Cavalcanti (eds.). Fundamentos para a coleta de germoplasma vegetal. EMBRAPA Recursos Genéticos e Biotecnologia, Brasília.

Wanderley, M.G.L., Shepherd, G.J. \& Giulietti, A.M. 2001. Flora Fanerogâmica do Estado de São Paulo. v. 1. FAPESP/Hucitec, São Paulo.

Wanderley, M.G.L., Shepherd, G.J. \& Giulietti, A.M. 2002. Flora Fanerogâmica do Estado de São Paulo. v. 2. FAPESP /Hucitec, São Paulo.

Wanderley, M.G.L., Shepherd, G.J. \& Giulietti, A.M. \& Melhem, T.S. 2003. Flora Fanerogâmica do Estado de São Paulo. v. 3. FAPESP/RiMa, São Paulo.

Wanderley, M.G.L., Shepherd, G.J., Melhem, T.S. \& Giulietti, A.M. 2005. Flora Fanerogâmica do Estado de São Paulo. v. 4. FAPESP /RiMa, São Paulo.

Wanderley, M.G.L., Shepherd, G.J., Melhem, T.S. \& Giulietti, A.M. 2007. Flora Fanerogâmica do Estado de São Paulo. v. 5. Secretaria de Meio Ambiente, Instituto de Botânica, FAPESP, São Paulo.

Wanderley, M.G.L., Shepherd, G.J., Melhem, T.S., Giulietti, A.M. \& Martins, S.E. 2009. Flora Fanerogâmica do Estado de São Paulo. v. 6. Secretaria de Meio Ambiente, Instituto de Botânica/ FAPESP, São Paulo.

Wanderley, M.G.L., Shepherd, G.J., Martins, S.E., Estrada, T.E.M.D., Romanini, R.P., Koch, I., Pirani, J.R., Melhem, T.S., Harley, A.M.G., Kinoshita, L.S., Magenta, M.A.G., Wagner, H.M.L., Barros, F., Lohmann, L.G., Amaral, M.C.E., Cordeiro, I., Aragaki, S., Bianchini, R.S. \& Esteves, G.L. 2011. Checklist das Spermatophyta do Estado de São Paulo, Brasil. Biota Neotropica 11(1a).

Wanderley, M.G.L., Shepherd, G.J., Melhem, T.S., Giulietti, A.M. \& Martins, S.E. 2012. Flora Fanerogâmica do Estado de São Paulo. v.7. Secretaria de Meio Ambiente, Instituto de Botânica/FAPESP, São Paulo.

Yamamoto, L.F., Kinoshita, L.S. \& Martins, F.R. 2005. Florística dos componentes arbóreo e arbustivo de um trecho da Floresta Estacional Semidecídua Montana, município de Pedreira, estado de São Paulo. Revista Brasileira de Botânica 28: 191-202.

Zenni, R.D. \& Ziller, S.R. 2011. An overview of invasive plants in Brazil. Revista Brasileira de Botânica 34: 431-446.

Ziller, S.R. 2001. Plantas exóticas invasoras: a ameaça da contaminação biológica. Ciência Hoje 30: 77-79. 